\title{
Ground Demonstration on the Autonomous Docking of Two 3U Cubesats Using a Novel Permanent-Magnet Docking Mechanism
}

\author{
Jing Pei* Luke Murchison†, Adam BenShabat $\ddagger$ Victor Stewart\$ \\ James Rosenthal, \\ Jacob Follman, Mark Banchy, and Drew Sellers ${ }^{\dagger \dagger}$ \\ NASA Langley Research Center, Hampton, Virginia, 23681 \\ Ryan Elandt, David Sawyer Elliott, Marc Choueirił; \\ Mechanical and Aerospace Engineering Department, Cornell University, Ithaca, New York, 14853 \\ Peter Finch \\ Mechanical and Aerospace Engineering Department, New York University, New York, NY, 10003
}

\begin{abstract}
Small spacecraft autonomous rendezvous and docking is an essential technology for future space structure assembly missions. A novel magnetic capture and latching mechanism is analyzed that allows for docking of two CubeSats without precise sensors and actuators. The proposed magnetic docking hardware not only provides the means to latch the CubeSats but it also significantly increases the likelihood of successful docking in the presence of relative attitude and position errors. The simplicity of the design allows it to be implemented on many CubeSat rendezvous missions. A CubeSat 3-DOF ground demonstration effort is on-going at NASA Langley Research Center that enables hardware-in-the loop testing of the autonomous approach and docking of a follower CubeSat to an identical leader CubeSat. The test setup consists of a 3 meter by 4 meter granite table and two nearly frictionless air bearing systems that support the two CubeSats . Four cold-gas on-off thrusters are used to translate the follower towards the leader, while a single reaction wheel is used to control the attitude of each CubeSat. An innovative modified pseudo inverse control allocation scheme was developed to address interactions between control effectors. The docking procedure requires relatively high actuator precision, a novel miminal impulse bit mitigation algorithm was developed to minimize the undesirable deadzone effects of the thrusters. Simulation of the ground demonstration shows that the Guidance, Navigation, and Control system along with the docking subsystem leads to successful docking under 3-sigma dispersions for all key system parameters. Extensive simulation and ground testing will provide sufficient confidence that the proposed docking mechanism along with the choosen suite of sensors and actuators will perform successful docking in the space environment.
\end{abstract}

${ }^{*}$ GNC Lead, Systems Analysis and Concepts Directorate, Vehicle Analysis Branch

${ }^{\dagger}$ Project Manager, Engineering Directorate, Structural and Thermal Systems Branch

${ }^{\ddagger}$ Mechanical Engineer, Engineering Directorate, Structural and Thermal Systems Branch

$\S$ Mechanical Lead, Engineering Directorate, Mechanical System Branch

IElectronics Engineer, Engineering Directorate, Electronic Systems Branch

$\|$ Electronics Engineer, Engineering Directorate, Electronic Systems Branch

** Software Engineer, Engineering Directorate, Flight Software Systems Branch

${ }^{\dagger \dagger}$ Software Lead, Engineering Directorate, Flight Software Systems Branch

$¥ \ddagger$ Graduate Student, Cornell University 


\section{Introduction}

CubeSat activities have become an important goal in NASA's technology roadmap. The agency recognizes the value of CubeSats as platforms for technology demonstrations and science missions. Assembly of multiple CubeSats in space into large structures such as a space telescope or solar panels could be tremendously beneficial for the science and engineering communities. Several research groups ${ }^{1-4}$ are currently tackling the challenges associated with autonomous rendezvous and docking of small-scale spacecraft on orbit. The On-orbit Autonomous Assembly of Nanosatellites (OAAN) team at NASA Langley was formed in response to the Announcement of Opportunity for the pilot Early Career Initiative from the Space Technology Mission Directorate (STMD) at NASA Headquarters. The idea to use permanent magnets to perform the alignment and locking of two CubeSats during the final phase of a docking maneuver in order to make the docking process robust in the presence of system uncertainties. A previous paper ${ }^{5}$ describes the development and analysis of the docking mechanism.

Ground testing of small satellites is not new and it offers a low cost platform for experimental validation of the proximity GNC (Guidance Navigation and Control) algorithms, sensors, and actuators. Several groups, ${ }^{6-8}$ including the well-known SPHERES team ${ }^{9}$ have successfully performed ground demonstrations. The intent of the ground demonstration is to verify key on-orbit functionalities within the constraints of the 3 Degrees of Freedom (DOF) (2 translation DOF, 1 rotation DOF) environment. However, unlike the other missions, OAAN system utilizes Carrier-Phase Differential Global Position System (CDGPS ${ }^{10}$ data to determine the relative position of the two CubeSats by measuring the phase difference of the GPS carrier wave at two locations, which provides relative position measurement accurate within $2 \mathrm{~cm}$. It is superior to visual navigation methods in terms of cost, complexity, size, mass, and power requirements. Extensive ground testing is required to estimate the errors in the sensors and actuators, and most importantly, to determine whether the GNC/docking subsystem designs are robust to such errors and allow for successful docking to take place.

Figure 1 shows the OAAN concept of operation and the axis system during the final docking phase for the on-orbit demonstration. Both the leader and follower CubeSats will be aligned with the $\mathrm{Z}$ body axis directed along the local v-bar direction of the LVLH (Local Vertical Local Horizontal) frame. The star tracker is aligned with zenith for the best view of the GPS constellation. The RCS (reaction control system) will bring the follower towards the leader at a closing velocity of a few $\mathrm{cm} / \mathrm{s}$. Once the CubeSats are within the basin of attraction of the magnetic docking subsystem, where magnetic forces and moments dominate the dynamics, feedback control will be turned off allowing for passive elimination of residual relative position and attitude errors. The CubeSats will then align and complete the docking process.

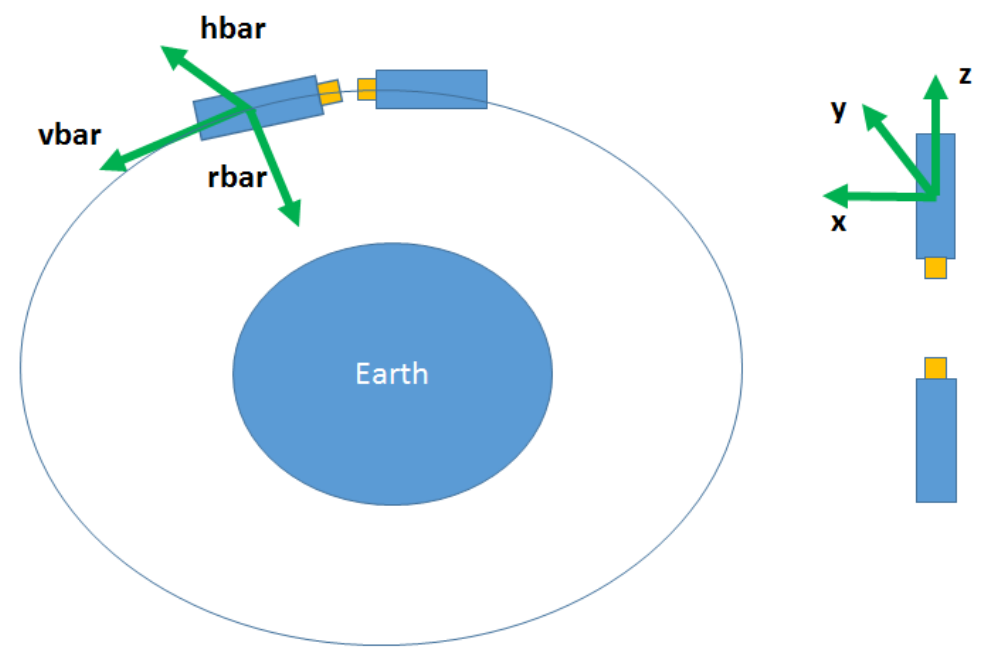

Figure 1. On-orbit Docking Concept of Operation

Figure 2 shows the OAAN concept of operation and the axis system for the ground demonstration of the docking phase. Both the leader and follower CubeSats will have their Z body axes aligned with the Z axis (long edge) of the testing table. Unlike the on-orbit mission, the Attitude Determination and Control 
System (ADCS) will only be using part of its functionality. The attitude sensors (star tracker, sun sensor, magnetometer) will be disabled, and only the rate gyros will be used to propagate the initial attitudes of the CubeSats. The RCS (same architecture as for the on-orbit mission) will bring the follower towards the leader. Once inside the magnetic docking subsystem's basin of attraction, any residual relative positional and attitude errors will be eliminated, and the docking process is considered complete once the docking subsystem latches the two CubeSats. The following terminology will be used to describe the relative separation between the CubeSats throughout this paper: $\mathrm{Z}$ describes the relative axial separation, $\mathrm{Y}$ describes the lateral separation, and $\mathrm{X}$ completes the right handed triad.

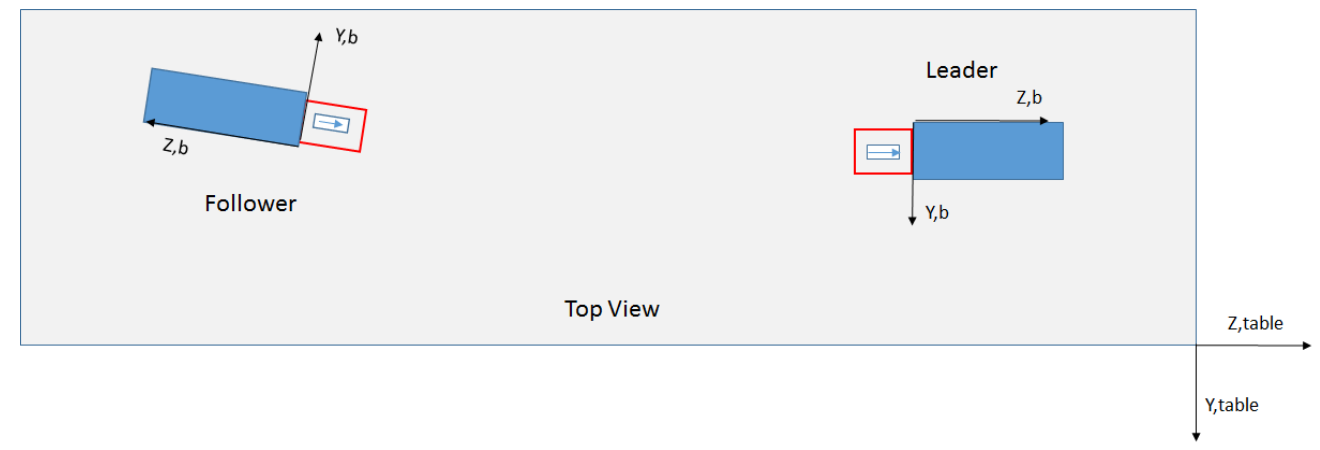

Figure 2. Ground Demo Docking Concept of Operation

Figure 3 shows the body frame of one test article (CubeSat + Low Drag Base). The Piksi ${ }^{10}$ (CDGPS) is mounted on the positive $\mathrm{X}$ face to ensure a clear view of the GPS constellation and the docking mechanism is located on the $-\mathrm{Z}$ face. The leader and follower test articles are both allowed to float on a granite table with minimal friction. The entire ground operation is designed to ensure maximum commonalities with the on-orbit mission. The common critical subsystems include: Attitude Determination and Control System (ADCS), Piksi (relative navigation sensor), magnetic docking system, and propulsion. The translational GNC architecture is similar between the two operations with differences in the controller gains and Kalman Filter implementations due to differences in the plant dynamics.

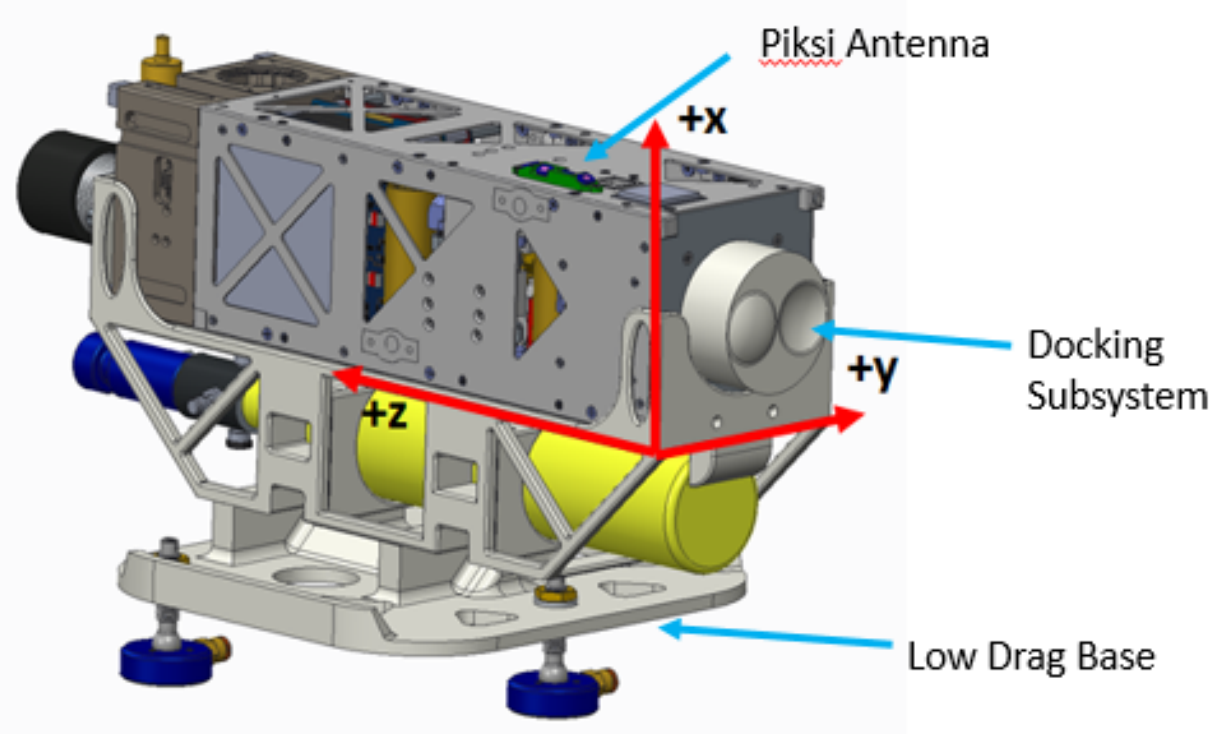

Figure 3. Test Article Body Frame

The remainder of this paper is organized as follows: Section II describes the major subsystems. This 
includes: cold gas propulsion, CDGPS, ADCS, and the magnetic docking subsystem. Section III describes the experimental test bed and the prototypes of the CubeSats used for the ground demonstration along with several important parameters such as table levelness, friction, and the mass properties of the test article. Section IV describes the design of the translational loop including the baseline controller, Kalman filter, a novel control allocation scheme, and a novel thruster MIB (Minimal Impulse Bit) mitigation algorithm. This is followed by the attitude control loop design, and finally evaluation of the docking mechanism using an energy approach. Section V presents simulation results of the ground demonstration. Finally, Section VI summarizes the results and provides discussion for on-going work.

\section{Key Dynamics Models}

\section{A. Propulsion}

For translational control, a four cold gas thruster layout design is used such that the thrusters provide pure translational motion as shown in Fig 4. The thrusters are strategically placed at the corners of an imaginary tetrahedron (middle $\mathrm{U}$ of the $3 \mathrm{U}$ CubeSat) with the thrust vector directed towards the centroid of the CubeSat. During the on-orbit operation, the center of mass (CM) should not to vary by more than 1 $\mathrm{cm}$ from the centroid throughout the mission; as a result, disturbance torque from the thrusters would be

kept at an minimum. For the ground test, the inclusion of the low drag base means that the $\mathrm{CM}$ of the test article will not coincide with the $\mathrm{CM}$ of the CubeSat alone, disturbance torques due to $\mathrm{r} \times \mathrm{F}$ will arise. Fortunately for the ground test, only torque about the $\mathrm{X}$ body frame is of significance. Therefore, as long as the z-cm of the test article is close to that of the CubeSat by itself, the ADCS is capable of rejecting any disturbance torque that may occur. Other factors that could result in disturbance torques are thrust vector misalignment and thruster mounting error (due to hardware imperfections).

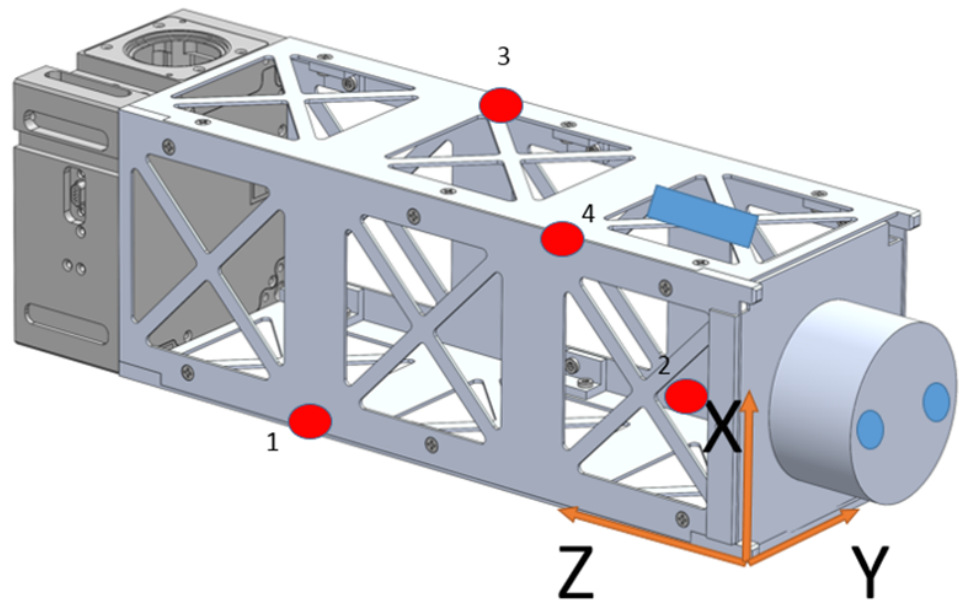

Figure 4. Thruster Layout

Theoretical thrust of each thruster is $0.3 \mathrm{~N}$ with a target throat diameter of around $0.8 \mathrm{~mm}$. In order to test the behavior of the thruster cluster, a 3-axis test stand was constructed. Details of the experimental setup and post processing steps can be found in Ref. ${ }^{11}$ The test setup enables the measurement of all three forces and moments. For a given run during the characterization test, the thruster combination ( 1 thruster, 2 thruster, 3 thruster firings, 14 total) and on-times (0.02 to 0.2 seconds) was selected at random. The random approach was taken to mitigate systemmatic errors such that gradual reduction in the upstream tank pressure during the test which affects the impulse delivered. For a given thruster combination, a nonlinear least squares approach was used to determine the best estimated thrust vector direction $\hat{r}$ and impulse vs. on-time data. Multiple characterization tests were performed to ensure consistency from test to test.

Thruster minimum impulse bit (MIB) is a major factor in determining the precision and accuracy that the translational control loop could achieve. ${ }^{12}$ The definition of MIB used in this project is: 1) minimal 
impulse that could be measured reliably, 2) above the MIB, the impulse vs. time should behave linearly. For a rendezvous and docking mission, it is desireable to keep the MIB at a minimum. Due to the relative small nozzle throat diameters, a tradeoff in the thruster design is being able to keep the throat diameter small enough to reduce the MIB and large enough such that the test data is repeatable. It was observed during testing that once the design throat diameter drops below a certain threshold, the test data becomes unreliable.

Figure 5 shows impulse measured vs. thruster on-time plot of three separate experimental tests intended to quantify the performance of an individual thruster. The dots represents the test points and the solid lines present least square fits through each data set. The average of the least-square fits was used to come up with a mean model for simulation. For thruster \#1, the MIB is approximately $0.022 \mathrm{~N}-\mathrm{s}$ and the nominal thrust is around $0.3 \mathrm{~N}$. Thrusters \#2,3, and 4 (not shown here), exhibit similar characteristics.

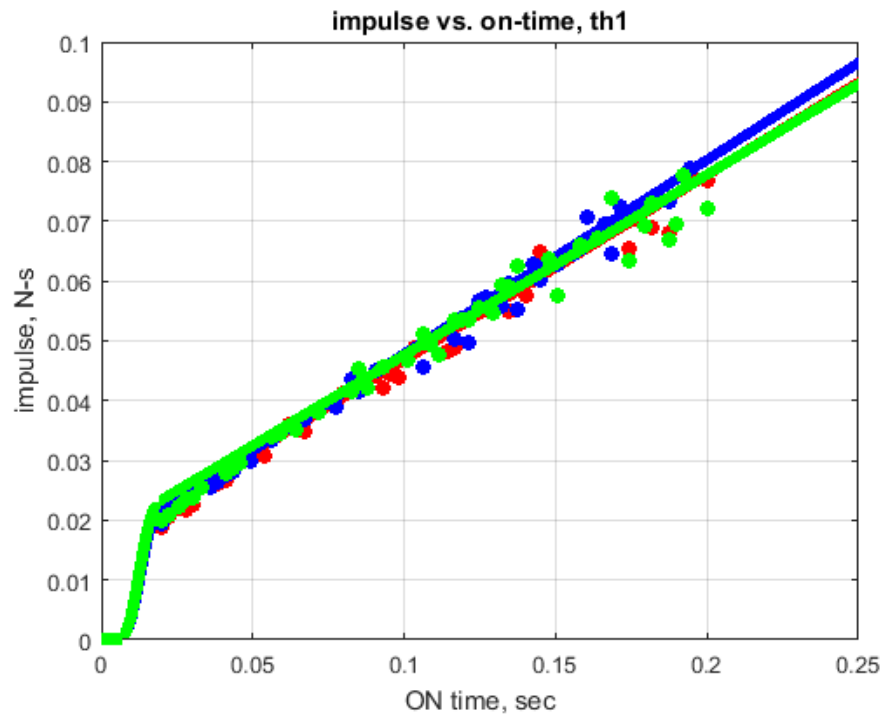

Figure 5. Impulse vs. Time, Thruster \#1

Table 1 compares the best estimated $\hat{r}$ with the ideal $\hat{r}$, where $\hat{r}$ is the thrust unit vector. Due to manufacturing imperfections (3D printing, installation, etc), the best estimated thrust vector directions deviate by 15 to $20 \mathrm{deg}$ from the theoretical tetrahedron configuration. Given the $\hat{r}$ and impulse vs. on-time data, a force vs. on-time model can be generated for each thruster firing combination. Figure 6 is a plot of the three-axis force vs. on-time model for thruster \#1. The initial deadzone during the first 0.005 seconds is associated with opening of the thruster valve. The sharp transient in the following 0.015 seconds is a typical behavior exhibited with cold gas thrusters. After 0.02 seconds, the thrust delivered is constant with on-time.

Table 1. Thrust Vector Ideal vs. Best Estimated

\begin{tabular}{ccccccc}
\hline \hline Thruster & X ideal & Y ideal & Z ideal & X BE & Y BE & Z BE \\
\hline 1 & 0.4652 & 0.5443 & -0.6981 & 0.3578 & 0.7788 & -0.5152 \\
2 & 0.4652 & -0.5443 & 0.6981 & 0.4779 & -0.6446 & 0.5968 \\
3 & -0.4652 & -0.5443 & -0.6981 & -0.3734 & -0.7671 & -0.5216 \\
4 & -0.4652 & 0.5443 & 0.6981 & -0.3993 & 0.7044 & 0.5868 \\
\hline \hline
\end{tabular}



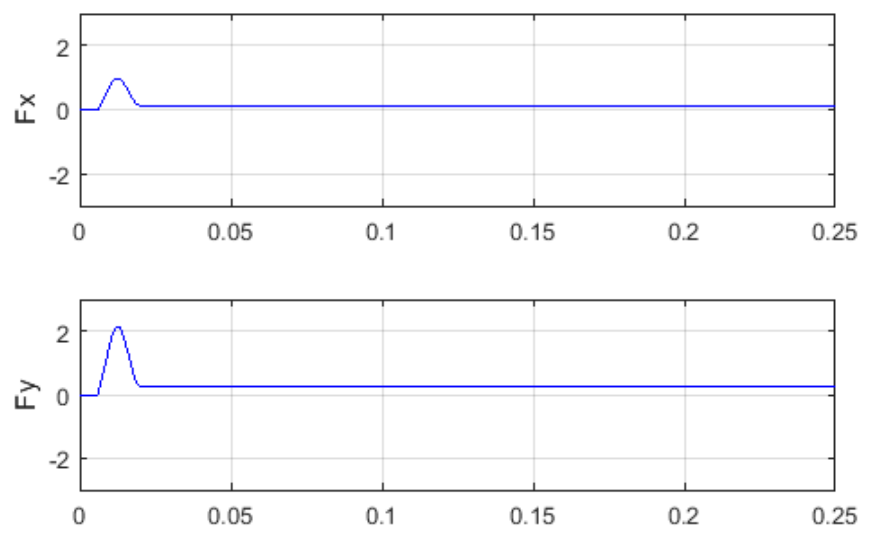

Force 1 thruster firing, th1

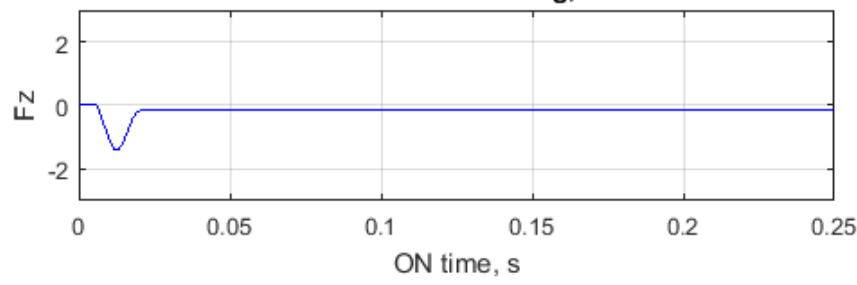

Figure 6. Force vs. Time model, Thruster \#1

Figure 7 shows impulse measured vs. thruster on-time plots for a two-thruster firing combination. Due to the piping layout and tank design, it was observed that the net impulse delivered when two thrusters are firing is not a sum of the individual thruster firing results (same $\hat{r}$ but impulse magnitude is off by a scale factor depending on the firing combination). This phenomenon was also observed in the pre-regulator pressure readings, as a drop in pressure immediately after a firing is more significant when more than one thruster is firing.

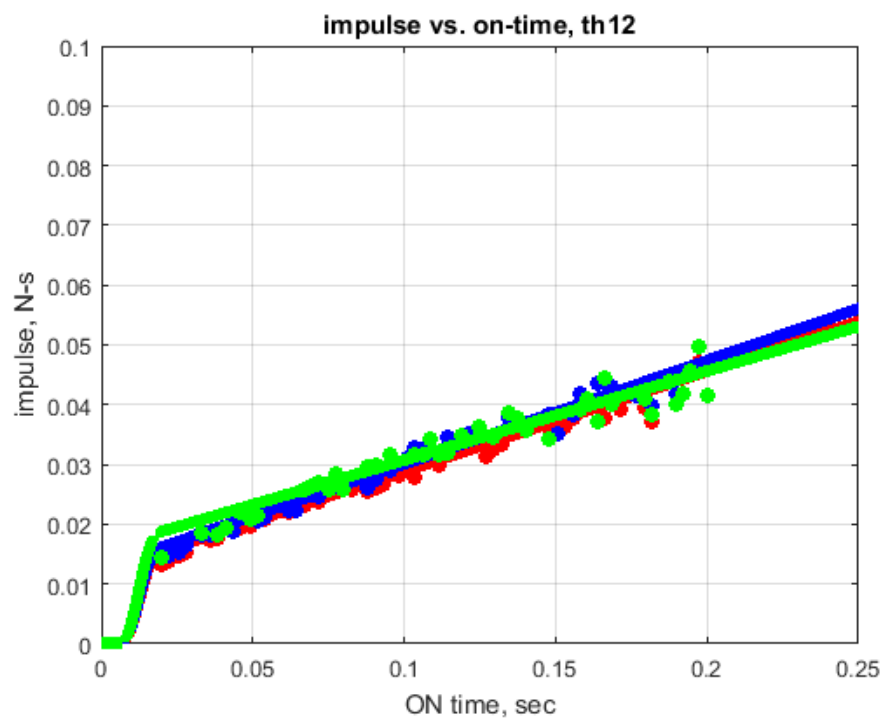

Figure 7. Impulse vs. Time, Thruster \#1,2

This same trend holds for three thrusters firing except the deviation in the impulse magnitude compared to the sum of individual thrusters firing is even more significant compared to two thrusters firing. This 
known performance deficiency creates additional complexity from a modeling and simulation perspective as a separate force vs. on-time model (similar to Figure 6) needs to be created for each thruster firing combination (14 total). Moreover, this known interaction between control effectors creates extra complexity from a control allocation standpoint. Discussions of the control allocation scheme is presented in Section IV.

\section{B. Relative Navigation Sensor}

Due to size constraints, cost, and simplicity considerations, CDGPS was chosen as the relative navigation sensor and only relative position measurements are communicated between the leader and follower CubeSat. Visual navigation methods such as using lasers and camera were deemed out of the project scope. CDGPS $^{13,14}$ allows for a few cm relative positioning accuracy when a RTK (real time kinematic) solution or a lock have been achieved between two units. CDGPS has been used use for precise CubeSat formation flight missions such as the CANX missions in 2014. ${ }^{15}$ In theory, the accuracy of the CDGPS should be adequate for a docking mission as well. The Swift Navigation Piksi ${ }^{10}$ is the commercial CDGPS hardware unit that was selected for OAAN. The Piksi units require one external antenna that faces away from Earth and toward the GPS satellites. A pair of units is required to obtain a relative navigation solution. One is designated as the rover and the other as the base. Once a RTK solution has been achieved the two units it will output a relative position and velocity vector between the two phase centers of the Piksi GPS antenna in the local North-East-Down (NED) or the Earth-Center-Earth-Fixed (ECEF) frame.

Data obtained by the Piksi includes the following errors: 1) Random noise. This error is not driven by a stochastic process and changes for each measurement. In the navigation software the noise is smoothed with a Kalman filter (assumes Gaussian distribution). 2) Bias error from GPS satellite constellation geometry. Docking success is sensitive to this slow moving stochastic error and tests indicate that this error could be as large as a few cm. 3) Error from incorrect Integer Ambiguity Problem (IAP) solution. The third error can range from a few $\mathrm{cm}$ to tens of meters in relative position. It can occur both suddenly during operation or arise during initialization of the Piksi hardware. Due to its rare occurrence, it is not considered part of the nominal docking scenario. Extensive static testing has been performed to obtain an initial estimate of the measurement noise covariance and bias error. These results are shown in this section.

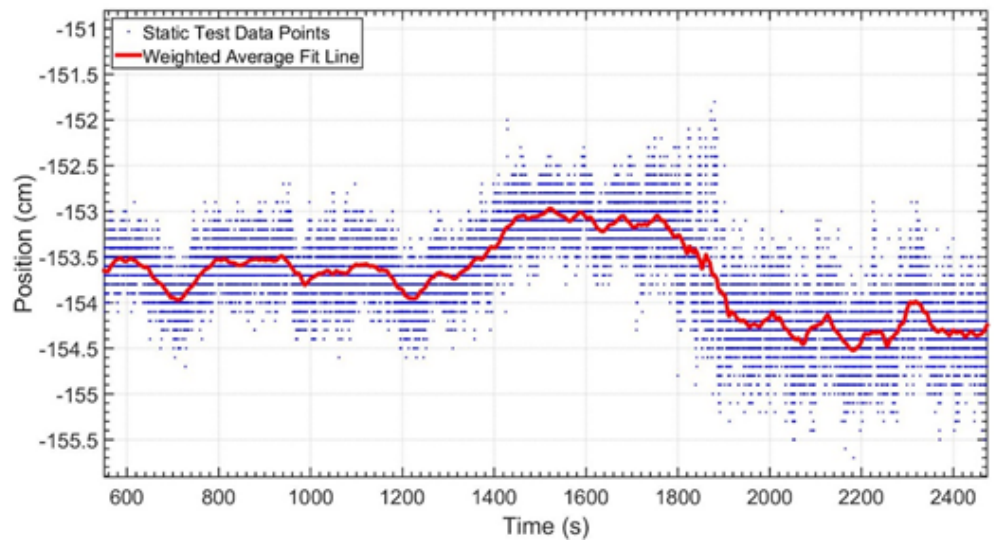

Figure 8. Sample Static Test

Figure 8 is a sample data set from a static test showing the east component of the NED relative position vector. Tests were performed in a wide open field that is free of obstacles to eliminate any errors due to multipath. The true distance was determined via a tape measure $(153.5 \mathrm{~cm})$, the blue dots are the measurements, and the red line is the weighted average. It is apparent from this plot that there are two noise components: 1) white noise, 2) slow moving stochastic bias. The residuals between the running average fit line and actual data points were used to determine the white noise. The amplitude of the running average fit line away from the true value represents the stochastic bias. Both of these parameters have means close to zero. The bias is slow moving and equally likely to be larger than the true value than it is to be smaller. To measure the stability of the bias, the mean and the standard deviation of the absolute value of the change in the amplitude is computed at each time step, which is selected as 25 seconds. Table 2 tabulates the standard 
deviations of the white noise and the bias stability. The errors (bias and noise) are largest in the Down direction, which is consistent with manufacturer specifications.

Table 2. CDGPS Error Parameters from Static Tests $1 \sigma$

\begin{tabular}{cccc}
\hline \hline Error Type & North & East & Down \\
\hline White Noise (cm) & 0.43 & 0.325 & 0.87 \\
Bias Stability (cm/time step) & 0.48 & 0.36 & 0.96 \\
\hline \hline
\end{tabular}

Figure 9 is a representative model of the Piksi error used in the simulation. The model consists of two sources of noise: 1) slow varying stochastic noise (red line) about the mean (green line), 2) white noise (blue dots) about the red line. The stochastic noise model had a time constant of 25 seconds. The bias model keeps track of a cumulative probability density function of the moving average line given the bias error; therefore, if the bias creeps towards the thresholds $(+/-6 \mathrm{~cm})$, then the tendency for it to keep growing in that direction reduces proportionally. At any time step, the movement of the moving average line is sized by a distribution based on the stability parameters. The model can be also be scaled by a gain factor or the time constant can be increased to affect the variability of the error signal and used during dispersion analysis.

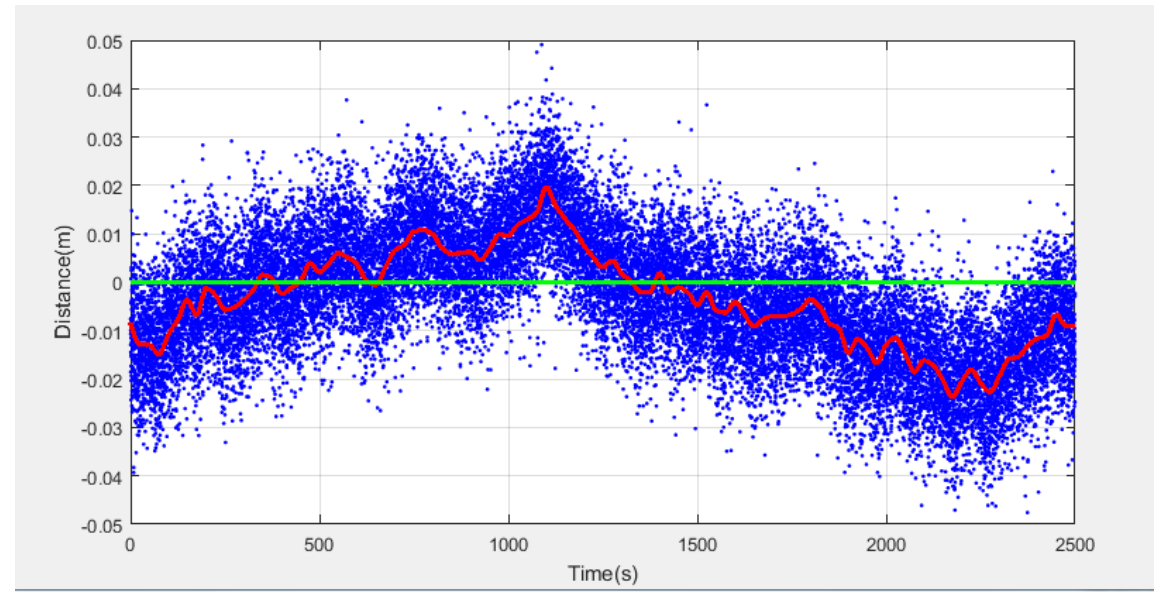

Figure 9. Sample Piksi error signal model

\section{Attitude Determination and Control}

The Attitude Determination and Control System (ADCS) is provided by Blue Canyon Technologies (BCT). The $\mathrm{XACT}^{16}$ unit is a fully assembled commercial off the shelf product with flight heritage. The XACT provides precise three-axis attitude control using reaction wheels, magnetic torque rods, and integrated control algorithms. For attitude sensors, it contains a star tracker, sun sensor, and magnetometer. The star tracker is used during fine pointing mode operations.

For the ground demonstration, only the gyroscope, accelerator, and one reaction wheel will be used. Figure 10 is a block diagram showing how the XACT unit is to be used. The coupled navigation-controlactuation loop inside the XACT would need to be broken for the ground experiment. At the beginning of a single trial, the user will specify a valid attitude (quaternion) for the leader and follower CubeSats (align the positive or negative $\mathrm{Z}$ body axis with the $\mathrm{Z}$ axis of the table). During the trial, the XACT will operate in the IMU-only mode that propagates the attitude of the CubeSat based on rate measurements. The attitude and rate errors would be fed into the attitude controller that is external of the XACT. The output of the controller (torque command) would then control the single reaction wheel inside the XACT. Section IV describes the reaction wheel dynamics and the design of the external attitude controller. 


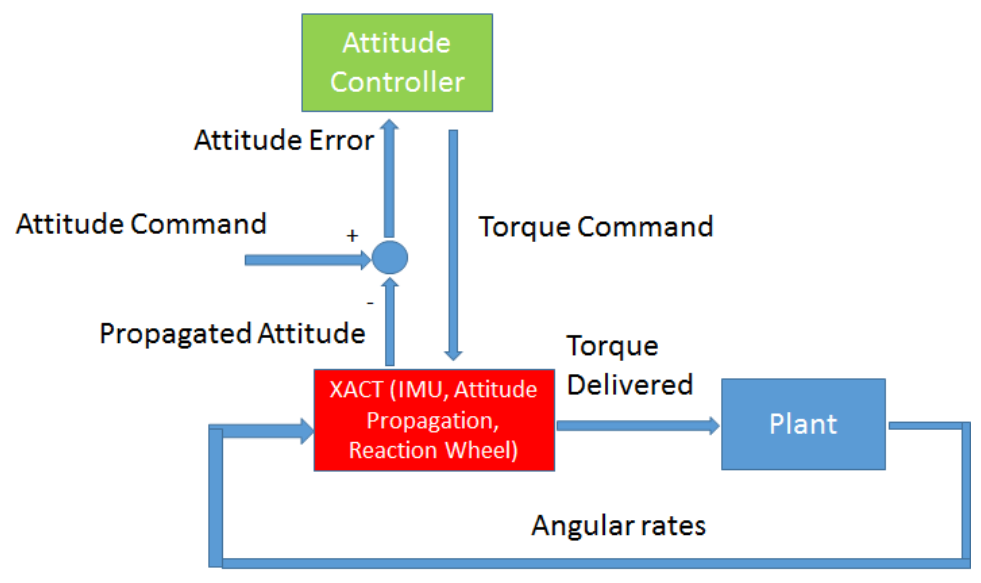

Figure 10. XACT: Ground Operation Model

Since the attitude of the test article is maintained by integrating the gyro measurements, error would build up over time due to random walk errors. Figure 11 is a plot provided by the vendor showing attitude error over time for the IMU-only operation (obtained through lab experiments). The maximum run time for each ground demonstration experiment is 300 seconds, therefore the attitude error should not exceed the $p m 2$ degrees bound. Table 3 contains the vendor specified IMU specs. The bias error is the key contribution to the secular drift shown in Fig. 11.

Table 3. XACT IMU Specs

\begin{tabular}{cc}
\hline \hline Spec & value \\
\hline internal update rate & $250 \mathrm{~Hz}$ \\
bias & $1.45 \mathrm{e}-5 \mathrm{rad} / \mathrm{s}$ \\
angle random walk & $0.0017 \mathrm{rad} / \mathrm{sqrt}(\mathrm{hr})$ \\
\hline \hline
\end{tabular}

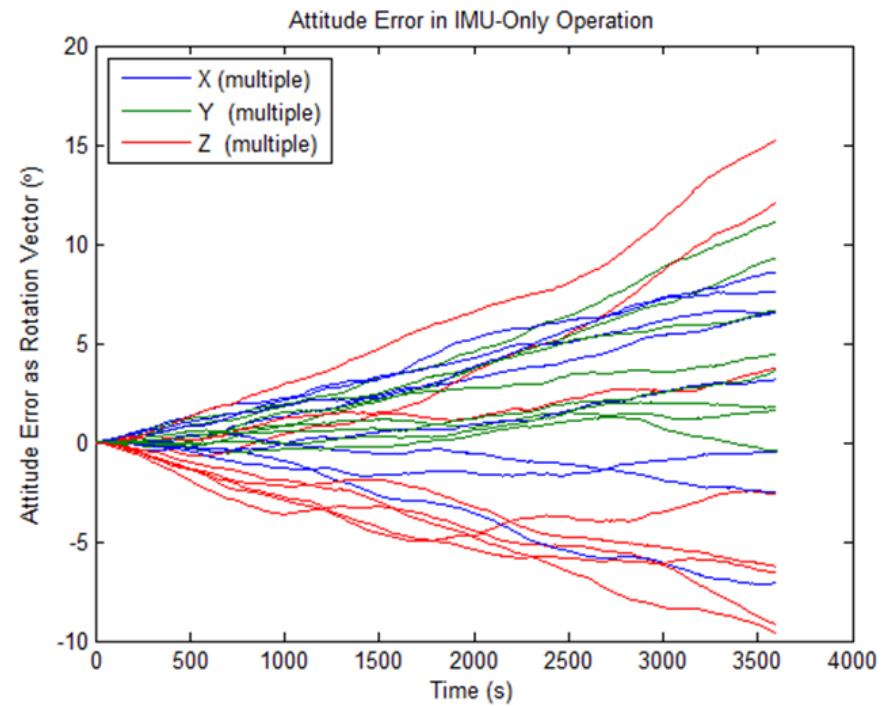

Figure 11. Attitude error: IMU only model

Table 4 shows the accelerometer specifications. The internal update rate of the accelerometer is $250 \mathrm{~Hz}$. An moving average FIR (Finite Impulse Response) filter is used to downsample the output to a $5 \mathrm{~Hz}$ signal 
which would be used by the Kalman filter. During each run, the turn-on bias will be estimated and removed.

Table 4. XACT Accelerometer Specs

\begin{tabular}{cc}
\hline \hline Spec & value \\
\hline internal update rate & $250 \mathrm{~Hz}$ \\
dynamic range & $+/-3 \mathrm{~g}$ \\
turn-on bias & $8 \mathrm{mg}$ \\
bias stability & $0.1 \mathrm{mg}$ \\
velocity random walk & $0.04 \mathrm{~m} / \mathrm{s} / \mathrm{sqrt}(\mathrm{hr})$ \\
\hline \hline
\end{tabular}

\section{Magnetic Docking Subsystem}

It is desirable from simplicity and a pure docking success standpoint to use a single large permanent magnet per docking subsystem. Doing so would make the system most robust to residual relative errors in the attitude and position loops. Practical constraints such as volume, mass, and the impact a strong electromagnetic field on other subsystems provides a hard limit on the size of the magnet. However from an attitude control perspective, a single magnetic dipole on board the CubeSat is problematic because a disturbance torque will be generated due to its interaction with the magnetic field of the Earth shown in Eq. $1^{12}$

$$
\overrightarrow{\tau_{d}}=\vec{M} \times \vec{B}
$$

where $\vec{M}$ is the net magnetic dipole moment of the docking subsystem and $\vec{B}$ is the Earth's magnetic field. A 0.5 inch cuboid magnet would generate a disturbance torque that is greater than the available control torque from both the reaction wheels and the magnetic torque rods. Due to this constraint, the docking subsystem is required to have a nominally zero net dipole moment. The next best choice is to have a pair of magnets per docking subsystem with the dipoles having opposite directions in the $\mathrm{Z}$ axis of the body frame. Figure 12 shows a schematic of the docking system. The capture range associated with this design is smaller compared to the single dipole design due to the existence of unstable equilibrium conditions in which the two docking subsystems would repel each other.

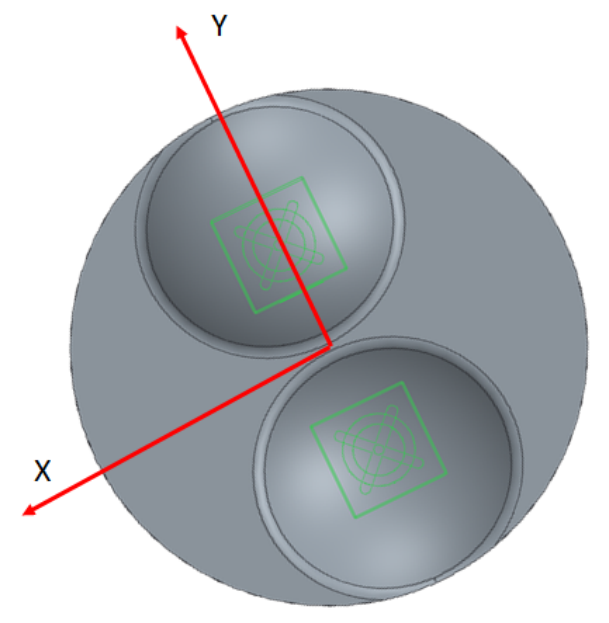

Figure 12. Magnetic Docking Subsystem Schematic

Yung, et ${ }^{17,18}$ provide analytical expressions for the force and moment produced by two magnetic dipoles and are provided in Eq. 2 and Eq. 3, respectively. These expressions are used to approximate the capture volume $^{5}$ between the docking subsystems and in the GNC simulations. Here $\hat{r}$ is the unit position vector from dipole a to dipole $\mathrm{b}$ and $\hat{m}$ is the magnetic dipole moment unit vector. It is important to note here that $\tau_{a b}$ is NOT equal to $-\tau_{b a}$. 


$$
\begin{gathered}
\vec{F}=\frac{3 \mu_{o} m_{a} m_{b}}{4 \pi|\vec{r}|^{4}}\left[\hat{r}\left(\hat{m}_{a} \cdot \hat{m}_{b}\right)+\hat{m}_{a}\left(\hat{r} \cdot \hat{m}_{b}\right)+\hat{m}_{b}\left(\hat{r} \cdot \hat{m}_{a}\right)-5 \hat{r}\left(\hat{r} \cdot \hat{m}_{a}\right)\left(\hat{r} \cdot \hat{m}_{b}\right)\right] \\
\vec{\tau}_{a b}=\frac{\mu_{o} m_{a} m_{b}}{4 \pi|\vec{r}|^{3}}\left[3\left(\hat{m}_{a} \cdot \hat{r}\right)\left(\hat{m}_{b} \times \hat{r}\right)+\left(\hat{m}_{a} \times \hat{m}_{b}\right)\right]
\end{gathered}
$$

The analytical force and moment expressions were calibrated via $\mathrm{COMSOL}^{19}$ and experimental testing. Comparison between COMSOL results and the simulation model governed by Eq. 2 and Eq. 3 is critical in understanding how well the analytical model predicts the actual magnetic forces and moments. The difference in the force becomes more pronounced at small separation distances which agrees with the assumptions that are associated with the analytical model. In the GNC simulation, a $20 \%$ knockdown factor is used for the magnetic force and moment model for conservatism.

\section{Experimental Setup}

\section{A. Environment}

The air bearings ${ }^{20}$ (which the test articles will sit on top of) have a friction coefficient of 1e-5. There are no differences between static and dynamic coeffients of friction so the stick-slip issue is completely eliminated. Friction in the air bearings is a function of air shear, so at zero velocity the friction is zero. A relay model $^{21}$ is adequate to capture first order effects and was chosen as the friction model for the simulation. A GPS compatible shelter was built to house the setup to eliminate any wind disturbances which would be significantly greater than the capabilities of the propulsion subsystem. The ground demonstration test bed is a $4 \mathrm{~m} \times 3 \mathrm{~m}$ granite table provided by MGL Marble \& Granite company. This serves as the floating base for the leader and follower test articles. Survey points were taken throughout the table to measure the local levelness. A least-square fit was applied to the data to obtain a contour of the table levelness as shown in Fig. 13. It is apparent that there are local minina and maxima (peaks and valleys) throughout the contour map. To approximate the disturbance forces acting on the test article due to gravity, the local gradients of the contour map were computed and stored as a lookup tables in the simulation. The peaks and valleys were qualitatively verified by placing the test article at specific locations on the table and observing its natural motions.

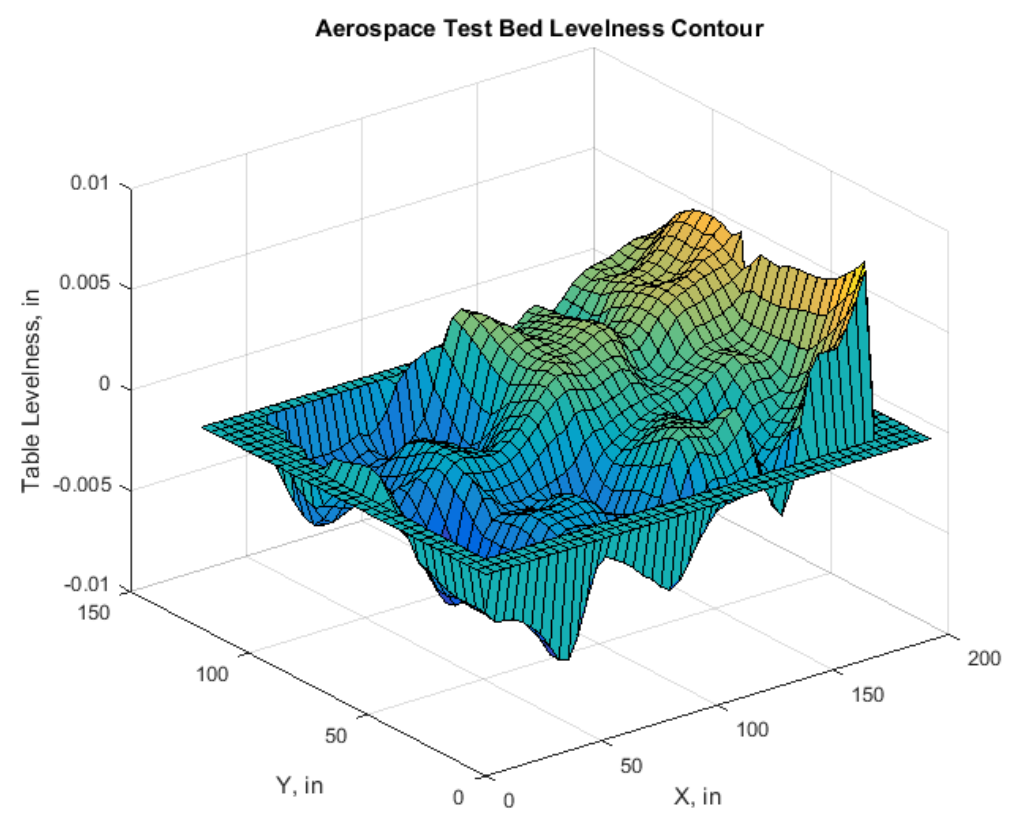

Figure 13. Test Bed Levelness Contour 


\section{B. Simulator}

Figure 3 is an illustration of the test article. The actual CubeSat sits on top of a low drag base that is capable of floating on the granite surface. The low drag base consists of: three air bearings, compressed $\mathrm{CO}_{2}$ cartridge, pressure gauge, and the support chassis that the CubeSat sits on. It is apparent from Table 5 that the mass properties of the test article will be different compared to the CubeSat by itself. Two important parameters that impact performance are: 1) Thrust vectors from the 4 thruster layout will not pass through the center of mass (CM) of the test article. Thus, disturbance torques will result and the attitude control should be designed such that it can reject such disturbances without saturation of the single reaction wheel. 2) Inertia about the $\mathrm{X}$ body axis of the simulator is significantly greater than the Cubesat by itself.

Table 5. Mass Properties: Test Article vs. CubeSat

\begin{tabular}{ccc}
\hline \hline Parameter & Test Article & CubeSat Alone \\
\hline Mass, kg & 8 & 5 \\
$I_{x x}, \mathrm{~kg}-\mathrm{m}^{2}$ & 0.009 & 0.005 \\
$X_{c g}, \mathrm{~m}$ & 0.02 & 0.0505 \\
$Y_{c g}, \mathrm{~m}$ & 0.048 & 0.048 \\
$Z_{c g}, \mathrm{~m}$ & 0.2 & 0.15 \\
\hline \hline
\end{tabular}

\section{GNC Design}

\section{A. Overview}

Due to weight, size, and power constraints of a CubeSat compared to previous spacecrafts that have attempted autonomous rendezvous and docking, the design philosophy for the entire OAAN mission (including the ground demo) is to keep the GNC algorithms as simple as possible. Since the long-term vision is to dock multiple CubeSats and assemble large space structures, it is desirable to use the minimal suite of cost effective sensors that permit robust completion of the mission. The navigation filters were designed considering lessons-learned from past projects such as DART (Demonstration of Autonomous Rendezvous Technology) and OE (Orbital Express). ${ }^{22}, 23$ The GNC design draws from previous ground demonstrations work by Romano and etc. ${ }^{6-8}$ Figure 14 is a top level schematic of the overall GNC architecture.

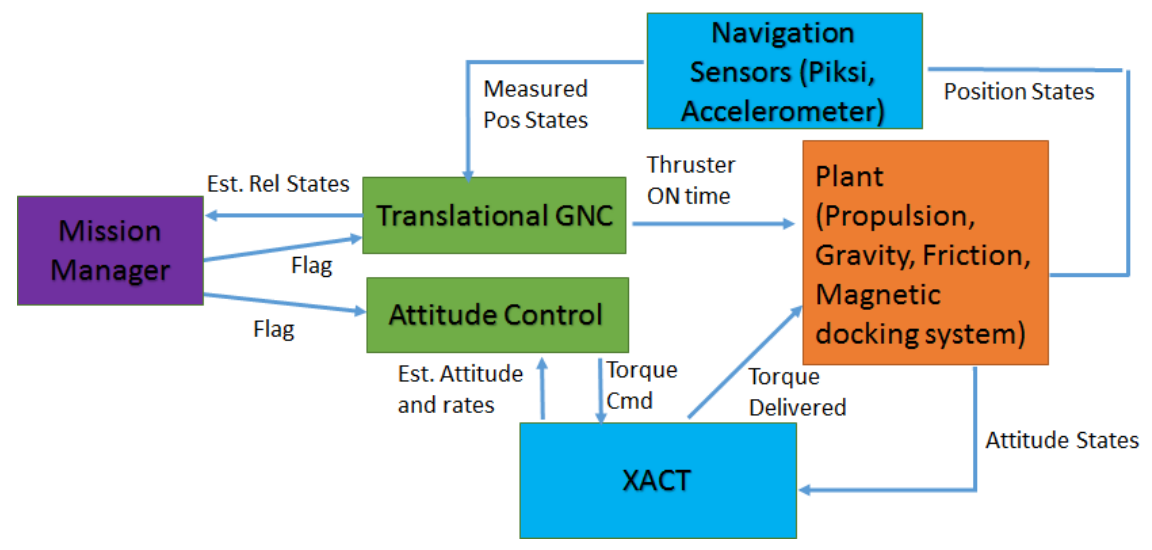

Figure 14. Top Level GNC Architecture

\section{B. Mission Manager}

A simple time-based mission manager was incorporated in the GNC software to determine when feedback control should be activated (flag $=1$ ). During first 15 seconds (feedback control off, flag $=0$ ), the navigation algorithm estimates the accelerometer turn-on bias and waits for Kalman filter innovation to settle down to 
a steady-state level. Thereafter, feedback control is activated in both the translational and attitude loops $(f l a g=1)$ until the follower is within the basin of attraction of magnetic docking subsystem $(\mathrm{Z} \leq 0.2 \mathrm{~m})$.

\section{Translational Loop}

Figure 15 shows a top level block schematic overview of the translational loop. Inside the navigation block, a Kalman filter is used to fuse relative position measurements from the CDGPS with accelerometer data from the XACT to come up with smoothed relative position and velocity estimates. In the event of a CDGPS false lock or slip ${ }^{13}$ or dropped signal, a false lock detection algorithm was developed to monitor the Kalman filter residuals and place the follower CubeSat in a safe state in case such an event occurs during flight or ground demonstration. If the anomaly occurs for less than 10 seconds or so, an option would be to have the controller act solely based on the Kalman filter propagated states. The guidance block is a lookup table that specifies the desired trajectory (relative position and velocity) of the follower. Due to imperfections in the propulsion subsystem design as discussed in Section II, an innovative modified linear allocator was designed to minimize the error between the impulse commanded at each control cycle and the actual impulse delivered. An optional reaction wheel momentum dumping routine was implemented in the event that the wheel saturates during ground testing (due to CM offset and thruster misalignment). The controller gains were designed so that there is adequate frequency separation between the translational and attitude loops. A linear controller was selected as the baseline with an optional augmenting adaptive portion to make the system more robust to highly uncertain parameters such as gravity disturbances from the unevenness of the flat floor. A novel MIB mitigation algorithm was designed to mitigate the undesirable effects of thruster minimal impulse bit. Finally, an energy $\operatorname{method}^{5}$ is used to analyze the basin of attraction of the magnetic docking subsystem.

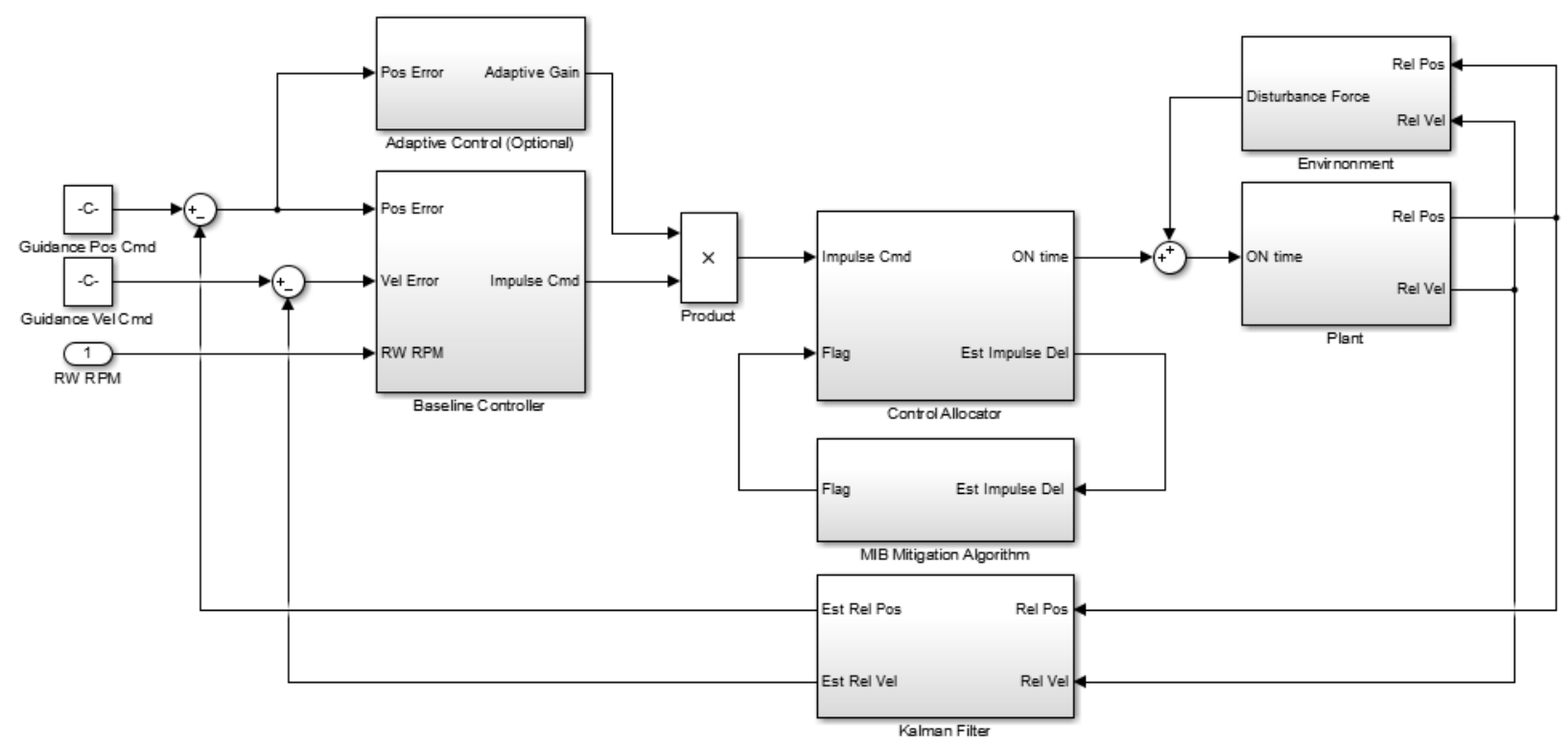

Figure 15. Top Level Translational Loop Block Diagram

\section{Control Allocation}

The control allocation scheme takes the acceleration (impulse) command at each control cycle $(3 \times 1$ vector $)$ and transforms it into thruster on-times $(4 \times 1)$. Since there are more control effectors than degrees of freedom, the solution to the control allocation problem is overdetermined. ${ }^{24,25}$ The objective is to find the on-time that would minimize the error between the commanded impulse and the impulse delivered at each control cycle subject to the effector constraints. In this case, the thruster on-time has to be between 0.02 to 0.2 seconds. Equation 4 illustrates the error minimization problem: ${ }^{26}$ given the observability and controllability matrices $C$ and $B$, find a vector $u$ such that 


$$
J=\left\|C B u-a_{d}\right\|
$$

is minimized, subject to $u_{\min } \leq u \leq u_{\max }$. Here $a_{d}$ is the desired impulse vector, $u$ is the thruster on-time.

A linear control allocation scheme via pseudo inverse is preferred due to its simplicity, ease of impementation, and compatability with linear analysis. At each control cycle, the allocation matrix maps the commanded impulse into thruster on-time. The thruster on-time is then converted to a pulse width modulation (PWM) signal (0 or 1$)$ with the width being the commaded on-time. The resolution of the commanded on-time in flight software is 0.001 seconds. Sidi ${ }^{12}$ offers an excellent explanation of the linear control allocation scheme for uni-directional Reaction Control System (RCS). The $B$ matrix (composed of magnitude and direction of each individual thruster) maps the thruster on-time into $F_{c}$ (force command). The pseudo inverse of $B$ in return maps $F_{c}$ into thruster on-time. Since the thrusters are uni-directional, one of the resultant on-time from the pseudo inverse solution will be negative. Negative duty cycles cannot be physically realizable since the thrusters can not be activated for negative time (thrusters are not bi-directional). As a result, one of the rows in the pseudo inverse matrix is ignored to make the algorithm operational.

The pseudo inverse algorithm has 2 major assumptions: 1) impulse vs. on-time curve is a straight line with the slope being the idealized thrust, 2) When two or more thrusters are firing simultaneously, the result force is the vector sum of the forces that are provided when the thrusters are firing individually. With the current propulsion design, neither one of the assumptions hold. Figure 16 is a plot of the actual impulse vs. on-time with the ideal impulse vs. on-time for thruster \#1. If the on-time is below the opening and closing time of the solenoids $(0.005 \mathrm{sec})$, the impulse delivered is zero. The time between the min on-time of the solenoids and when steady thrust is reached is considered the transient portion ( 0.005 to $0.2 \mathrm{sec}$ ). This is apparent in the sharp rise of the impulse vs. on-time plot and modeled as an initial hump in the force vs. on-time plot shown in Section II.

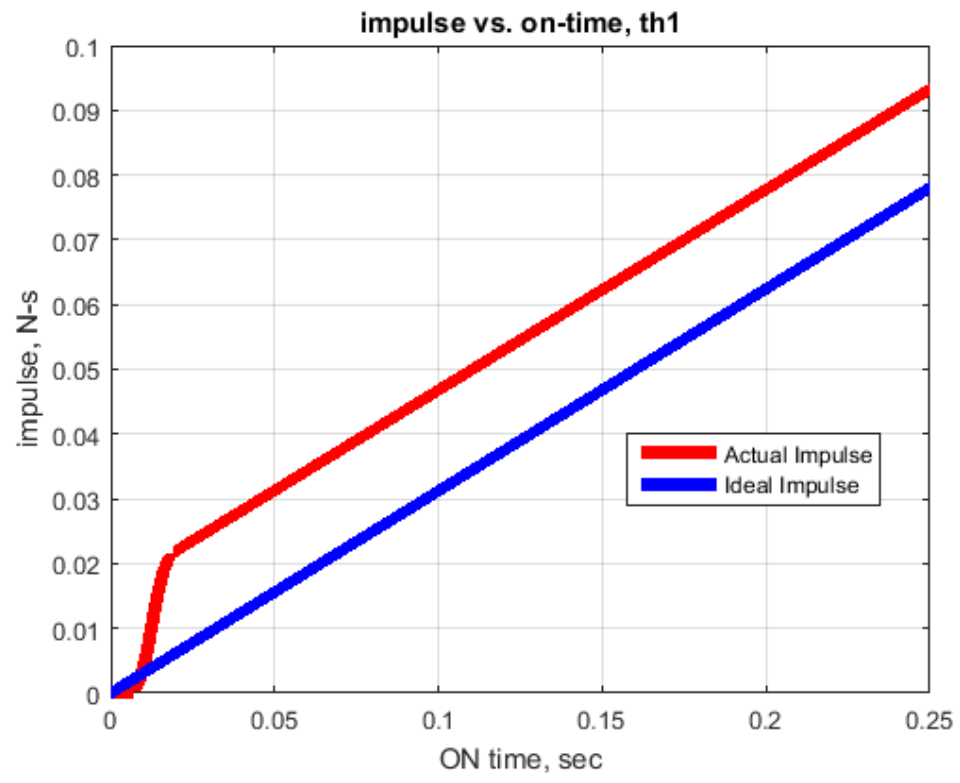

Figure 16. Actual and Ideal Impulse vs. on-time

Few works exist in the literature that address control effector interactions in the control allocation problem. ${ }^{27}$ A modified pseudo inverse algorithm was developed to take into consideration known control effector interactions and the nonlinear impulse vs. on-time characteristics. Figure 17 is a schematic of the algorithm. There are two key assumptions: 1) The thruster layout can achieve an arbiturary impulse vector in any direction and magnitude (limited by the on-time constraints) by firing the thrusters in three sequential stages. For instance, 3 thrusters firing, followed by 2 , then by 1 . Or 2 thrusters firing followed by 1 . The number of thrusters firing has to decrease monotonically and the on- time for the current stage must be greater than the one prior. With the thruster layout, there are 24 unique combinations in which the thruster firings could be made, subject to the constraints described. This is referred to as the truth table and shown in Table 6 . 
Here columns 2-4 represents the unique thruster firing combinations for stage 3, columns 5-6 for stage 2, and column 6 for stage 1. 2) The forces delivered for all 14 thruster firing combinations (3 thrusters, 2 thrusters, 1 thruster firing) are repeatable and can be superimposed linearly.

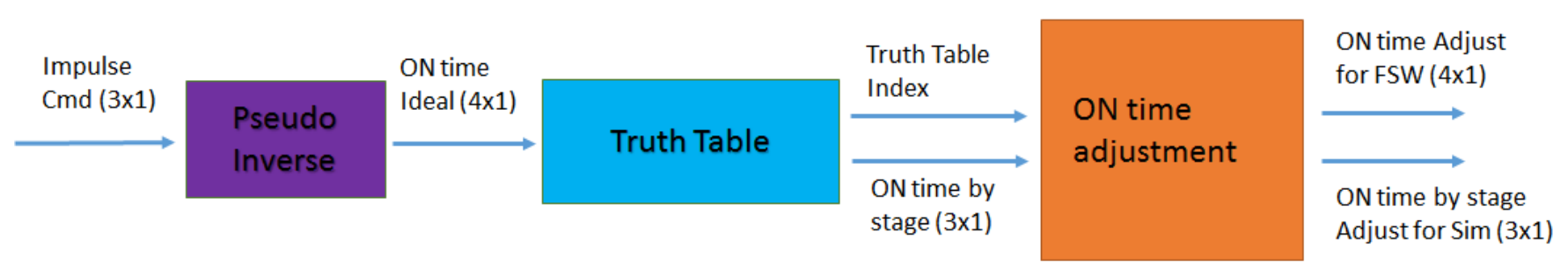

Figure 17. Control Allocation Schematic: Modified Pseudo Inverse

Table 6. Modified Psuedo Inverse Algorithm: Truth Table

\begin{tabular}{lllllll}
\hline \hline 1, & 2 & 3 & 4 & 3 & 4 & 4 \\
2, & 2 & 3 & 4 & 3 & 4 & 3 \\
3, & 2 & 3 & 4 & 2 & 4 & 4 \\
4, & 2 & 3 & 4 & 2 & 4 & 2 \\
5, & 2 & 3 & 4 & 2 & 3 & 3 \\
6, & 2 & 3 & 4 & 2 & 3 & 2 \\
7, & 1 & 3 & 4 & 3 & 4 & 4 \\
8, & 1 & 3 & 4 & 3 & 4 & 3 \\
9, & 1 & 3 & 4 & 1 & 4 & 4 \\
10, & 1 & 3 & 4 & 1 & 4 & 1 \\
11, & 1 & 3 & 4 & 1 & 3 & 3 \\
12, & 1 & 3 & 4 & 1 & 3 & 1 \\
13, & 1 & 2 & 4 & 2 & 4 & 4 \\
14, & 1 & 2 & 4 & 2 & 4 & 2 \\
15, & 1 & 2 & 4 & 1 & 4 & 4 \\
16, & 1 & 2 & 4 & 1 & 4 & 1 \\
17, & 1 & 2 & 4 & 1 & 2 & 2 \\
18, & 1 & 2 & 4 & 1 & 2 & 1 \\
19, & 1 & 2 & 3 & 2 & 3 & 3 \\
20, & 1 & 2 & 3 & 2 & 3 & 2 \\
21, & 1 & 2 & 3 & 1 & 3 & 3 \\
22, & 1 & 2 & 3 & 1 & 3 & 1 \\
23, & 1 & 2 & 3 & 1 & 2 & 2 \\
24, & 1 & 2 & 3 & 1 & 2 & 1 \\
\hline \hline & & & & & & \\
\hline \hline
\end{tabular}

The algorithm works as follows: 1) From the impulse command, determine the idealized thruster on-time based on standard pseudo inverse, 2) From the idealized on-time, sort the on-time by stages and find the corresponding index in the truth table, 3) Adjust the idealized stage on-time by the experimental impulse data (i.e Fig 16). Thruster on-time saturation limits apply: if the adjusted stage on-time is 0.02 sec or less set the stage on-time to 0 , if the adjusted stage on-time is greater than 0.2 set the stage on-time to 0.2 sec. 4) The adjusted on-time by thrusters $(4 \times 1)$ is passed on to flight software (FSW), the adjusted on-time by stage $(3 \times 1)$ is used for simulation purposes in which the force vs. on-time per stage is modeled as look-up tables. 


\section{Guidance}

The simplest guidance algorithm is to have the follower's controller regulate its position relative to the leader. Initially, the controller would see a large error in the axial $(\mathrm{Z})$ direction and the error would decrease as the follower manuevered its way towards the leader. Another approach is to have the guidance specify a desired approach profile in the axial direction (relative position and velocity) and have the controller follow that profile (Y channel would still be a regulator). This would eliminate issues such as control saturation (if the initial error is too large) or the need for gain scheduling. Figure 18 shows an example of a desired axial trajectory. The idea is to have the follower slow down as much as possible prior to reaching the basin of attraction of the magnetic docking subsystem.

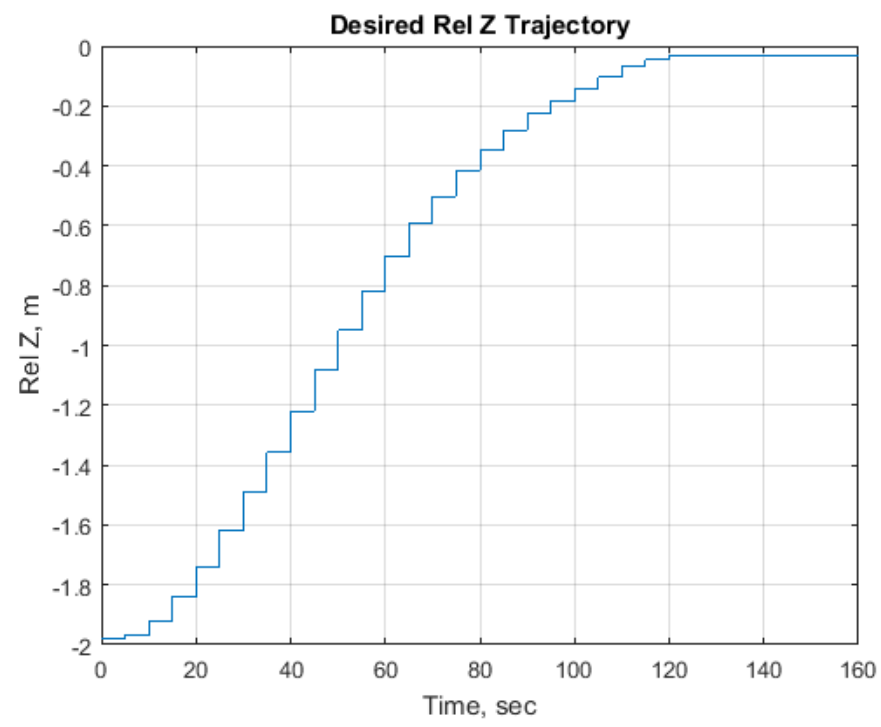

Figure 18. Sample Guidance Profile: Relative Z Position

\section{Controller Design}

For the in-space docking phase, a low bandwidth full state feedback docking controller about the linear Clohessy-Wiltshire equations ${ }^{28}$ was designed to bring the follower CubeSat towards the leader at a few $\mathrm{cm} / \mathrm{s}$. There have been numerous studies that explore docking controller strategies. ${ }^{29-32}$ A nonlinear phase plane controller also seemed to work well by giving the designer the option to choose the desired coast velocity, position and velocity dead bands etc. in each channel with the magnetic docking subsystem capture volume in mind. To keep as much commonalities as possible between the ground demo and the in-space GNC design, a linear controller is used for the translational loop with the dynamics being a double integrator. The two main objectives are: 1) keep the bandwidth low such that there is roughly an order of magnitude of frequency separation with the attitude loop, 2) make sure that the closed-loop poles of the second order system is well damped with $\zeta>0.7$. This would ensure that the follower approachs the magnetic basin of attraction with minimal residual linear velocity. 

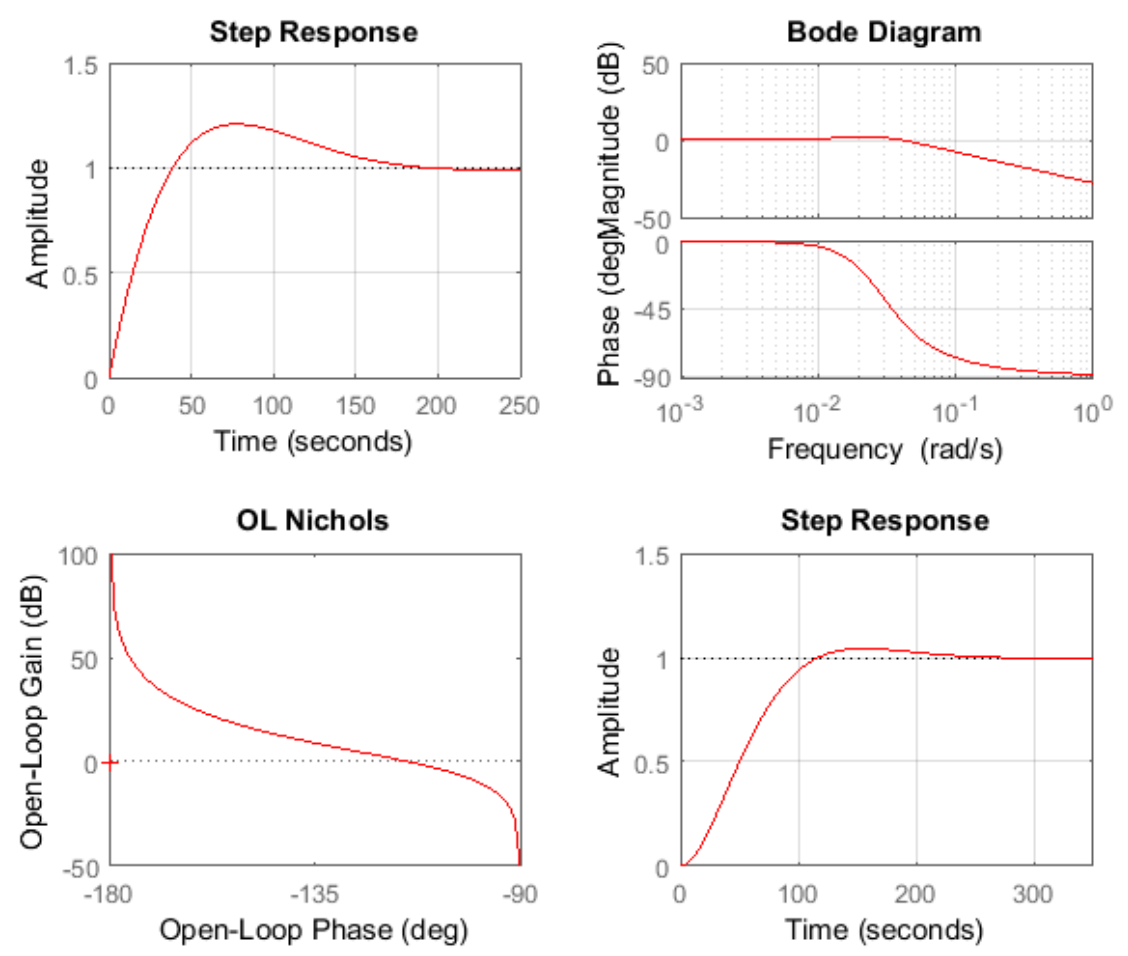

Figure 19. Linear Stability Analysis

Figure 19 presents LTI (Linear Time Invariant) plots of the $\mathrm{Y}$ and $\mathrm{Z}$ channels. The top left plot shows the closed-loop step response from position command to actuator output. Top right and bottom left plots are the Bode and Nichols plots of the open-loop transfer function. From the Nichols plot it is apparent that the closed-loop system is unconditionally stable with infinite gain margin (double integrator dynamics) with a phase margin of approximately 65 degrees at a gain crossover frequency of $0.007 \mathrm{~Hz}$. The bottom right plot is the closed-loop step response of the position command. A small integral gain was included to provide better performance with respect to disturbances such as table unevenness and friction. With the integral gain in the loop, the system has a gain margin of $6 \mathrm{~dB}$ and a reduced phase margin of 51 degrees.

\section{MIB Mitigation algorithm}

As mentioned in Section II the minimum impulse bit (MIB) associated with the thrusters acts similar to a deadzone (nonlinear element). The MIB is defined as the minimum impulse that the thrusters could reliably produce which corresponds to a minimum thruster on- time. To enforce this, logic inside the control allocation was incorporated such that if the commanded on-time is less than the minimum on-time then set the on-time to zero. This is equivalent to having a deadzone in the loop and makes the behavior of the linear controller less predictable. The basic concept of the MIB algorithm is as follows: keep track of whether an impulse has been applied to the vehicle during the current control cycle based on the commanded on-time (an accurate force vs. on-time model is kept inside the control allocation block). If the estimated impulse delivered approximates the impulse commanded then proceed with the next control update (flag $=$ 1). Otherwise $(\mathrm{flag}=0$ ), start accumulation of the commanded impulse as follows: Fcmd $=$ Fcmd,current + Fcmd,previous. This will continue until the impulse commanded is large enough to generate on-time commands that are greater than the minimal on-time. Figure 20 shows the block diagram illustrating this concept. 


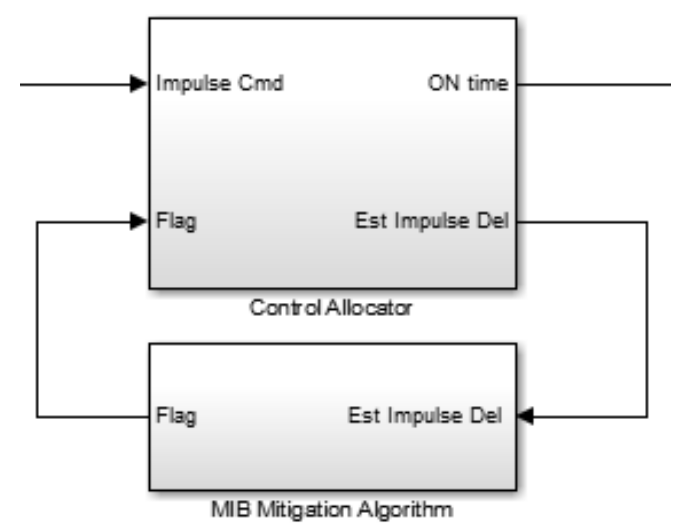

Figure 20. MIB compensation algorithm

\section{Navigation}

A linear Kalman filter ${ }^{33}$ is used to fuse the accelerometer data from the XACT and the position data from the CDGPS to come up with smoothed relative position and velocity estimates for the controller. The continuous A, B, C, D matrices of the Kalman filter are shown below. These are then converted to discrete time for real time implementation. The process noise, $Q_{d}$ and measurement noise covariances $R_{d}$ (discrete forms) were determined experimentally to be $0.002 \mathrm{~m} / \mathrm{s}^{2}$ and $0.003 \mathrm{~m}$. The accelerometer noise covariance determined is consistent with the random walk specs provided by the vendors shown in Table 4. Gaussian distributions are assumed for the noise covariance. Table 7 shows the sample times of the sensors, navigation, and control algorithms.

$$
A=\left[\begin{array}{ll}
0 & 1 \\
0 & 0
\end{array}\right] B=\left[\begin{array}{l}
0 \\
1
\end{array}\right] C=\left[\begin{array}{ll}
1 & 0 \\
0 & 1
\end{array}\right] D=\left[\begin{array}{l}
0 \\
0
\end{array}\right]
$$

Table 7. Sample Times

\begin{tabular}{cc}
\hline \hline Parameter & Ts (seconds) \\
\hline Accelerometer (raw) & 0.004 \\
Accelerometer (averaged) & 0.02 \\
CDGPS & 0.1 \\
Kalman Filter & 0.5 \\
Controller & 1 \\
\hline \hline
\end{tabular}

One subtlety to note here is that the accelerometer is not co-located with the test article CM. To be accurate, an state observer ${ }^{34}$ is required to estimate the acceleration at the CM. Sensitivity analyses were performed and results showed that the off-CM effects are negligible and should not affect performance of the GNC subsystem, hence it is not necessary to add complexity in the navigation algorithm. Equation 6 illustrates the sensed acceleration at a location on the body that's not collocated with the CG:

$$
\ddot{r}_{S}=\ddot{r}_{B}+\ddot{r}_{B S}+2 \omega \times \dot{r}_{B S}+\dot{\omega} \times r_{B S}+\omega \times \omega \times r_{B S}
$$

here $r_{B S}$ is the location of the accelerometer with respect to the test article CG and $\omega$ is the body frame angular velocity.

A false lock detection and compensation algorithm was developed as part of the navigation block in the event of a false lock or dropped signal. False Lock is also known as false slip, ? this occurs when the algorithm inside the GPS receiver incorrectly solves the integer ambiguity problem (IAP) and yields a solution that could be centimeters or tens of meters off from the truth (even though the lock flag $=1$ ). The dropped 
signal case is when the lock flag displays 0 even though a lock (flag $=1$ ) has already been established. The false lock detection algorithm compares the innovation, $y_{k}$ (measurement - estimate) to $S_{k}$, the state estimate covariance matrix. If a threshold is exceeded or if lock flag $=0$, then a false lock flag would be triggered, the Kalman gain would be set to 0 inside the Kalman filter routine and the controller would be operating solely based on the propagated states. A blending scheme then re-blends the measurements with the model estimates once the Piksi measurements can be trusted again. Figure 21 shows a flow diagram of the operational scenarios in the event of a false lock or dropped signal.

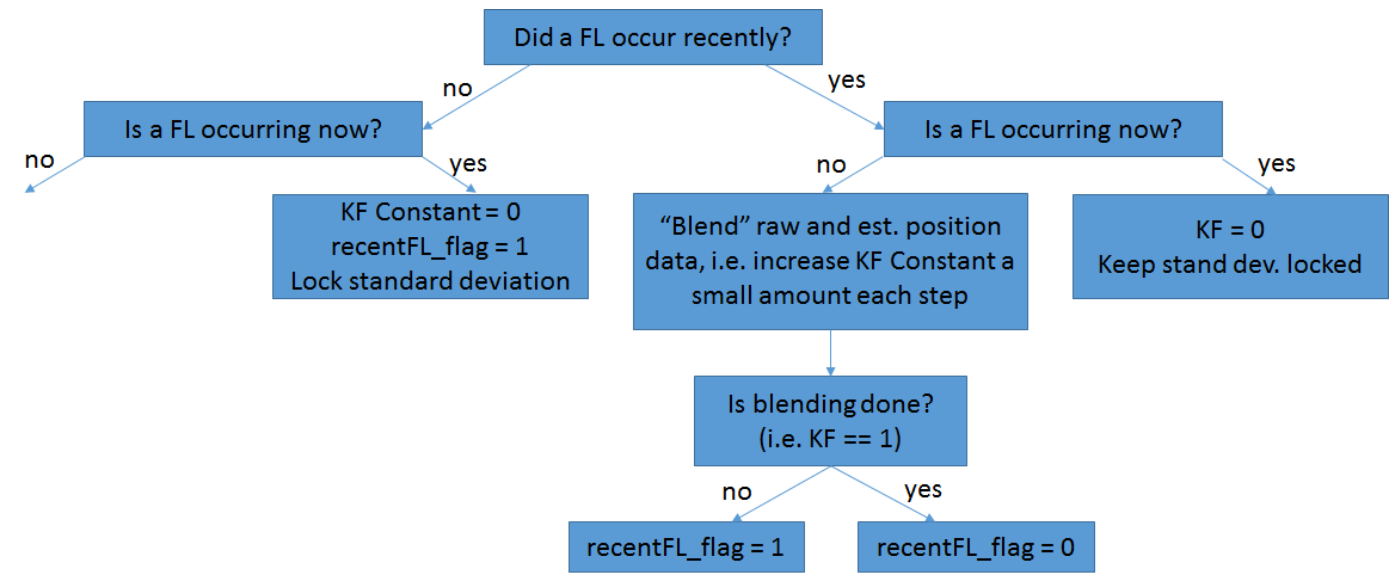

Figure 21. CDGPS False Lock Algorithm Flow Diagram

\section{Attitude Loop}

Figure 22 shows the block diagram of the attitude loop. As mentioned in Section II, the XACT GNC software is designed for an in-space mission. For the ground demonstration, a controller external to the XACT was designed to command the the single reaction wheel inside the XACT. The dynamics of the reaction wheel can be best approximated by the $200 \mathrm{~Hz}$ discrete transfer function model ${ }^{16}$ shown in Eq. 7. The command latency is between 5 to $205 \mathrm{~ms}$. The maximum torque of the wheel is $0.004 \mathrm{Nm}$ and decreases linearly (due to back-EMF) as the wheel approaches a top speed of $6000 \mathrm{RPM}$ (this latter effect is not modeled in the simulation).

$$
T(z)=\frac{0.4665}{z-0.5335}
$$

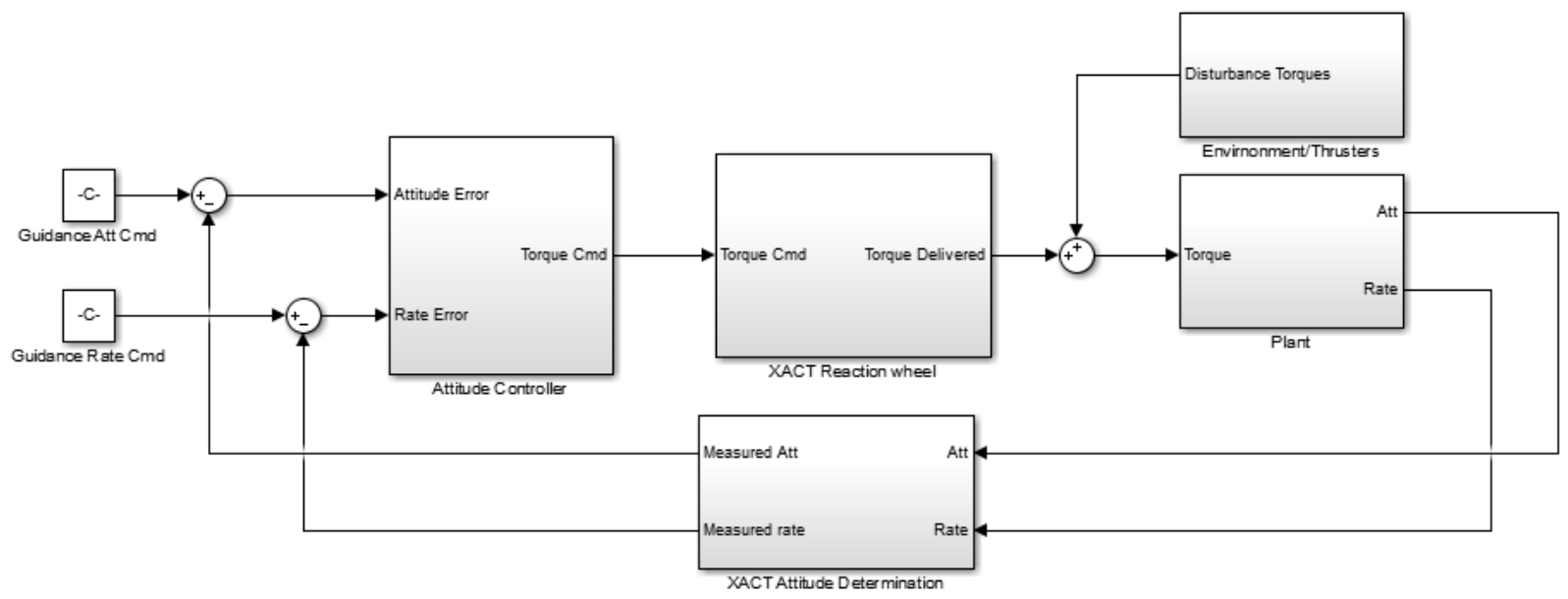

Figure 22. Attitude Loop Block Diagram 
Similar to the translation channel, a PD controller is used. Desired characteristics of the second-order closed-loop transfer function are as follows: 1) 10 times higher bandwidth than the translational loop, 2) well damped $(\zeta=0.7)$. Figure 23 presents stability plots of the attitude channel. The system is unconditionally stable with roughly 60 degrees of phase margin at a gain crossover frequency of $0.0748 \mathrm{~Hz}$.
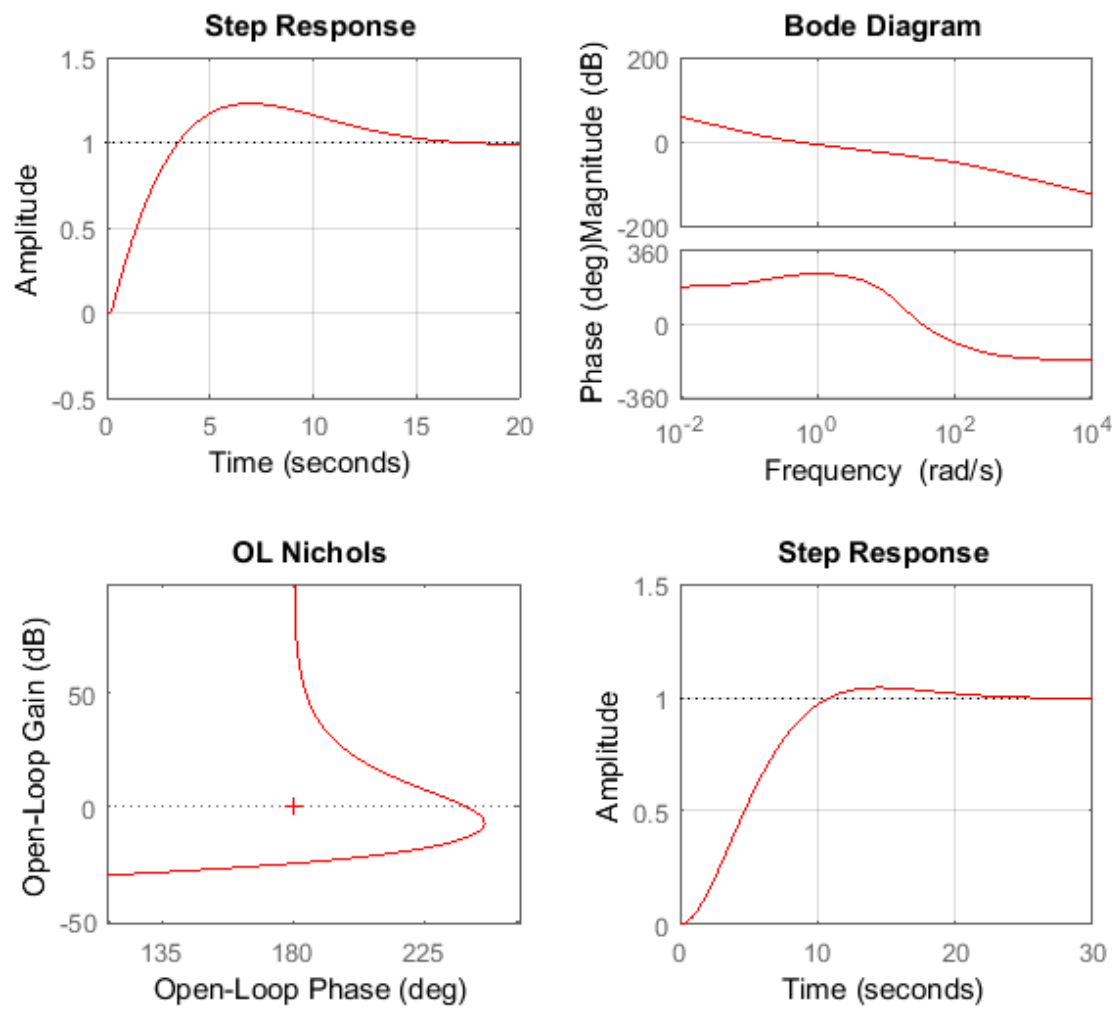

Figure 23. Linear Stability Analysis: Attitude Channel

Due to CM offset and thruster misalignment, the test article would experience a significant amount of disturbance torque during a given trial. A reaction wheel momentum dump option is incorporated in the event the reaction wheel is close to saturation. Depending on the direction of the wheel angular momentum buildup, either thrusters \#2 and \#4 will be used to dump the excessive moment. Thrusters \#2 and \#4 are most effective due the long moment arms. This action is undesireable when the CubeSats are close to docking.

\section{E. Docking Analysis}

A novel energy approach was used to optimize the virtual target location which is defined to be a point directly in front of the docking interface center of the leader CubeSat. In the ideal operational scenario, the GNC algorithm brings the follower CubeSat to a virtual target location directly in front of the leader CubeSat with zero kinetic energy; subsequently, feedback control is turned off and the magnetic potential gradually brings the two together. Docking is guaranteed in this ideal case because the kinetic energy will always be smaller compared to the potential energy at the target location. Figure 24 shows a schematic of the docking phase. The red dot indicates the virtual target location which the follower aims for and the cone represents the virtual basin of attraction associated with the magnetic docking mechanism. Refer to $\mathrm{Pei}^{5}$ for detailed discussion on the magnetic docking design and analysis. 


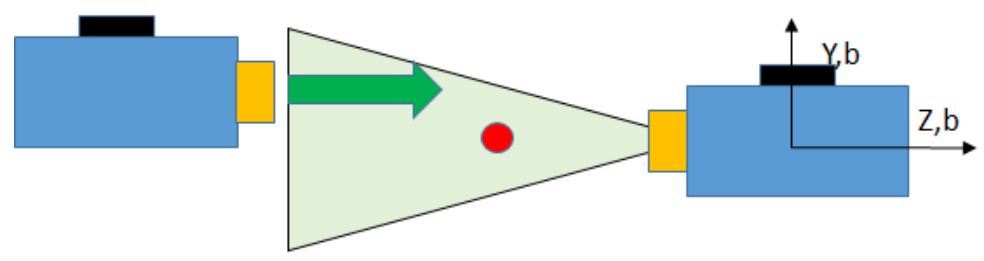

Figure 24. Docking Schematic

\section{Simulation Results}

Preliminary in-space simulation results suggest that at the beginning of the docking phase the CubeSats will be separated by some along-track distance (V-bar) with minimal separations in the cross-track (R-bar) and $\mathrm{H}$-bar directions. To replicate similar initial conditions, the baseline scenario for the ground demo is to have the CubeSats separated by $3 \mathrm{~m}$ in the axial (Z) direction with minimal lateral (Y) offsets. The attitudes of the test articles are such that their $\mathrm{Z}$ body axes are aligned with the long edge of the table with their docking interfaces pointed facing each other. Termination conditions in the simulation are: 1) Successful docking, when the offset between the centerlines of the docking interfaces (when touching) are within a circle of $1 \mathrm{~cm}$ radius. 2) Unsuccessful docking, when the follower overshoots the leader or when the magnetic docking subsystem generates large attitude motions in the CubeSats due to excessive misalignment error between the docking subsystems. Note: collision and contact dynamics are not modeled in the simulation. Due to the excessive gravity disturbance forces from table uneveness and its uncertain nature, simulations results presented in this section are separated into: 1) Table levelness not included, 2) Table levelness included. Table 8 is a comprehensive list of dispersion parameters used for the Monte Carlo simulations. The nominal values are shown in the middle column and the $1 \sigma$ dispersions are shown in the third column.

Table 8. Dispersions Parameters

\begin{tabular}{ccc}
\hline \hline Parameter & Nominal & Uncertainty $(1 \sigma)$ \\
\hline Thruster misalignment angle, deg & $10-20$ & 5 \\
Thruster mounting offset, $\mathrm{m}$ & 0 & 0.0015 \\
Thruster impulse magnitude, $\%$ & 0 & 5 \\
CDGPS bias (time varying), m & 0 & 0.02 \\
CDGPS noise, m & 0 & 0.003 \\
Accelometer noise, $\mathrm{m} / \mathrm{s}^{2}$ & 0 & 0.002 \\
Accelometer bias, m/s ${ }^{2}$ & 0 & $2.943 \mathrm{e}-4$ \\
Initial attitude misalignment, deg & 0 & 1.5 \\
Gyro drift, rad/s & 0 & $2.67 \mathrm{e}-5$ \\
Magnetic knockdown factor, $\%$ & -20 & 0 \\
IMU misalignment, deg & 0 & 1.5 \\
Avionic delay, ms & 25 & 0 \\
CG offset, m & 0 & 0.0033 \\
Inertia $I_{x x}, \%$ & 0 & 3 \\
Friction coefficient & $1 \mathrm{e}-5$ & 0 \\
\hline \hline
\end{tabular}

\section{A. Table Levelness not Included}

Figures 25 to 28 show plots of the follower and leader's states and actuator outputs for a nominal successful run. Figure 25 is a time history plot of the follower and leader's position in the frame of the flat floor. The green line represents the trace of the follower's docking interface center and the red dashed line represents the target location that is in front of the leader's docking interface. In this run, the CDGPS error is small and the follower enters the magnetic basin of attraction with a small attitude error offset in Y direction. The 
magnetic docking subsystem subsequently corrects for the position offset caused by the CDGPS bias, aligns the two CubeSats, and finally snaps them in place. Figure 26 shows the final offset between the docking interfaces once they are in contact. Monte Carlo simulation shows roughly 1 or 2 runs out of every 100 fail to meet the criteria. Robust docking is not possible (not shown here) without the aid of the proposed magnetic docking subsystem given the uncertainties in the system dynamics.
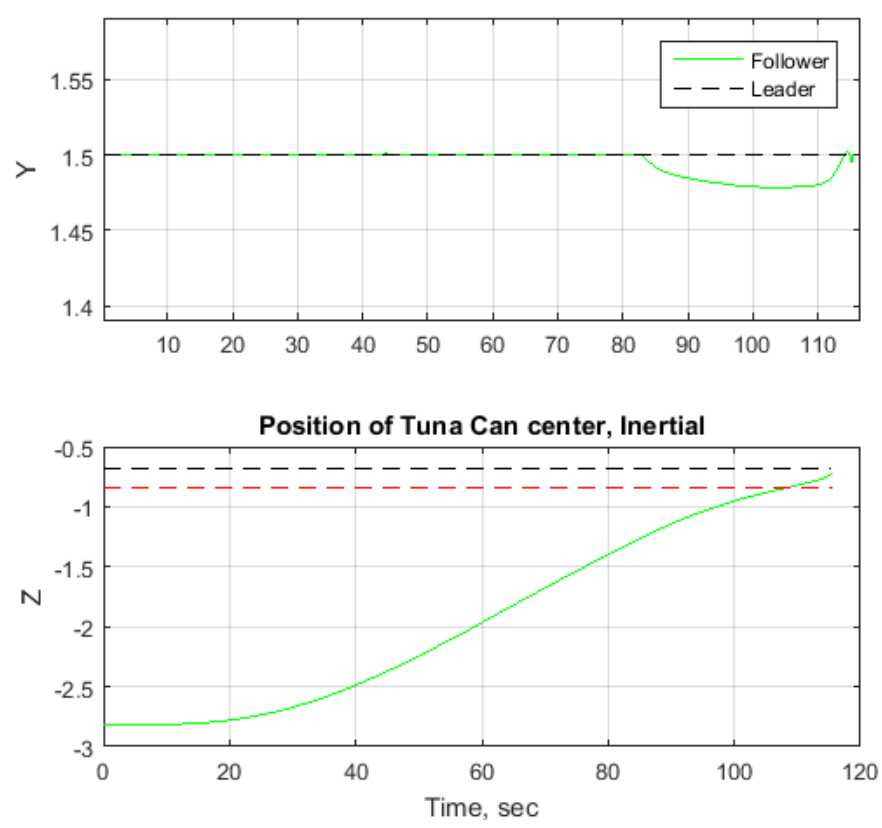

Figure 25. Nominal Successful Docking Example: Relative Position Time History

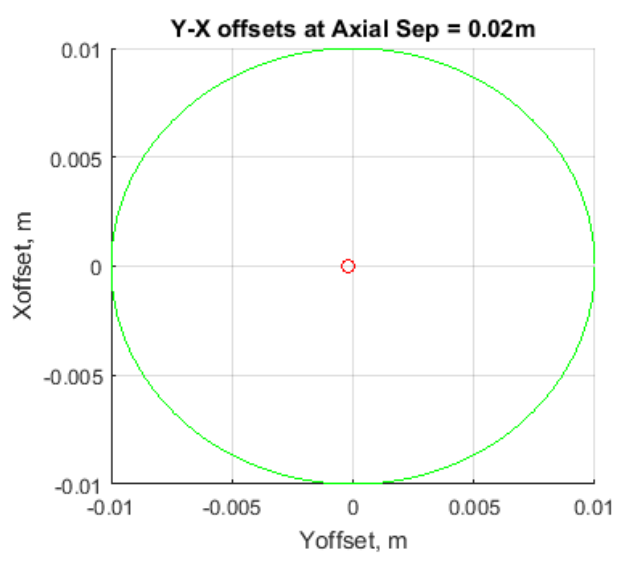

Figure 26. Nominal Successful Docking Example: Final Offset

Figure 27 shows a time history of the thruster duty cycle. During first 15 seconds, the thrusters are inactive such that the Nav algorithm can remove any accelerometer and IMU turn-on biases and wait for the Kalman filter to converge. Thrusters 1 and 3 then fire to propel the follower towards the leader. After T $=80 \mathrm{sec}$, thruster 2 and 4 then fire to slow down prior to reaching the target location. At approximatly $\mathrm{T}$ $=82 \mathrm{sec}$, the allocation scheme generates an on-time command such that only thruster 4 fires. This causes a slight offset in $\mathrm{Y}$ and a jump in the attitude error. The dV consumed is on the order of $0.06 \mathrm{~m} / \mathrm{s}$. 

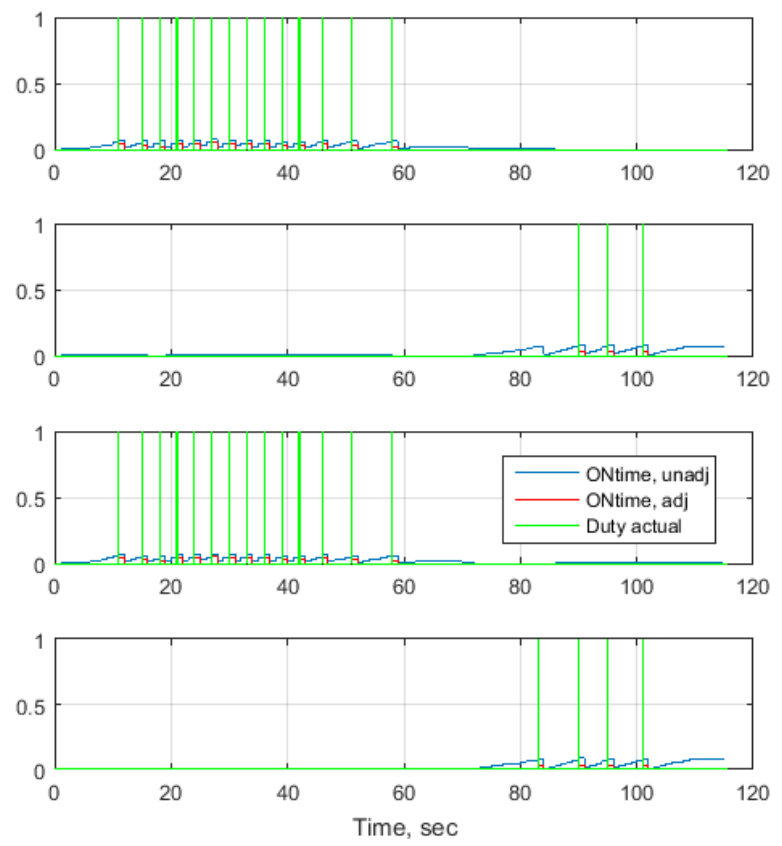

Figure 27. Nominal Successful Docking Example: Thruster Duty

Figure 28 show time history plots of the follower's attitude error in the X channel and wheel speed. The attitude error stayed below 1 deg throughout the run until the magnetic forces and moments align the two test articles and wheel speed stayed well within the saturation limits.
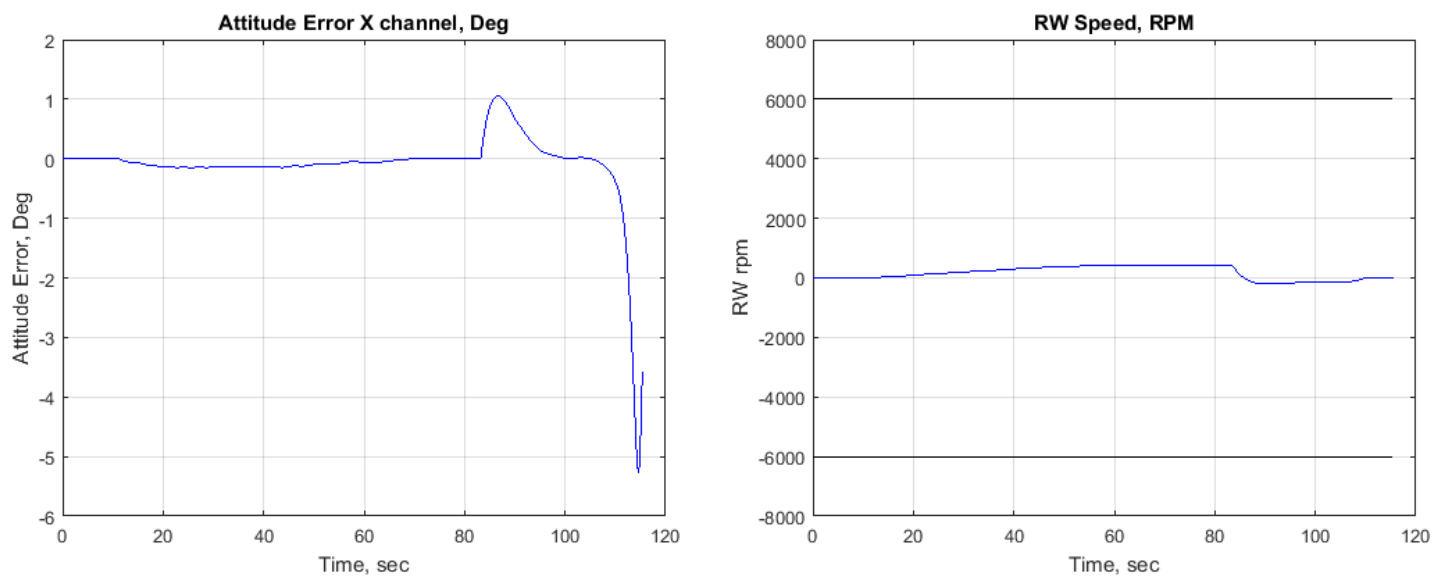

Figure 28. Nominal Successful Docking Example: Attitude Error and Reaction Wheel Speed

\section{B. Table Levelness Included}

Figures 29 to 33 show plots of the follower and leader's states and actuator outputs for a nominal successful run with table unevenness disturbance model included. The leader is assumed to be stationary and only allowed to rotate. As shown in Section III, the gravity disturbance forces due to table uneveness are significant and not uniform throughout the table. Moreover, the uncertainties associated with the survey points could be off by as much as \pm 0.004 inches. This presents a difficult challenge to the GNC design as a single set of gains is no longer robust depending on the initial location of the follower on the flat floor. Small adjustments to the gains need to be applied in order for successful docking to take place as the follower's starting location varies. Figures 29 and 30 are plots of the follower test article's docking interface center position time history 
for the baseline scenario. Figure 31 show the plot of the final offset between the docking interfaces.
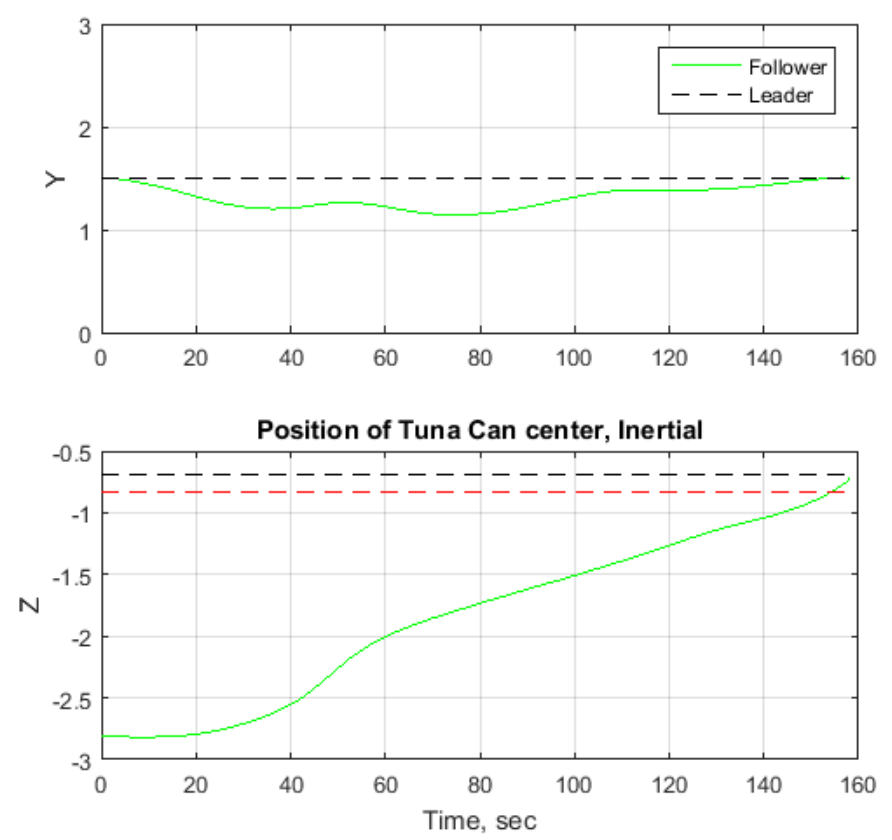

Figure 29. Nominal Successful Docking Example: Relative Position Time History

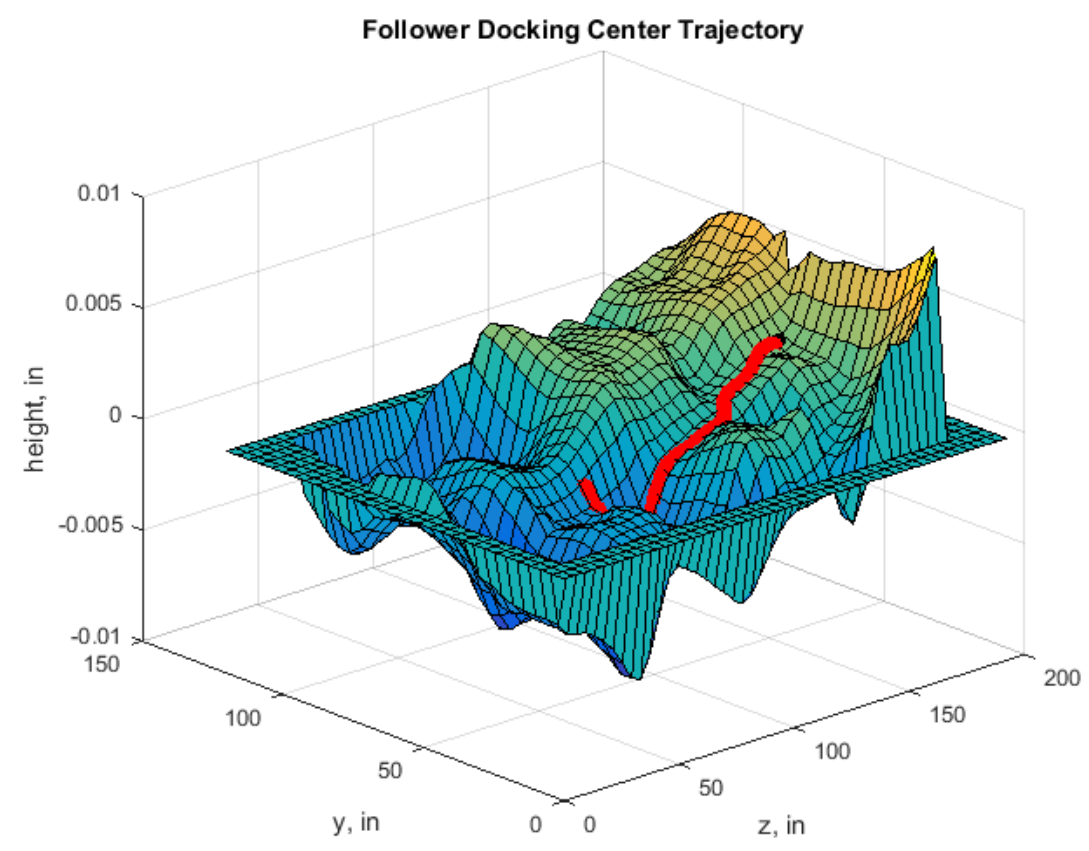

Figure 30. Nominal Successful Docking Example: Position Time History 


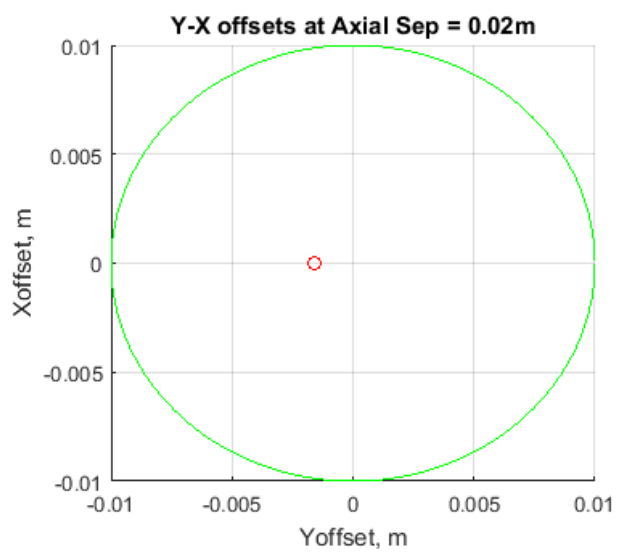

Figure 31. Nominal Successful Docking Example: Final Offset

It is apparent from the plots that the trajectory is significantly different compared to the case where table uneveness is not included. The desired small approach velocity in the axial direction is no longer exhibited (as the follower attempts to "climb over" over a ridge). Here the thruster forces are constantly applied to eliminate the disturbance forces due to gravity. Figure 32 shows the thruster duty cycles. The estimate dV is $0.15 \mathrm{~m} / \mathrm{s}$ which is almost 3 times greater compared to the same run with the table uneveness removed.
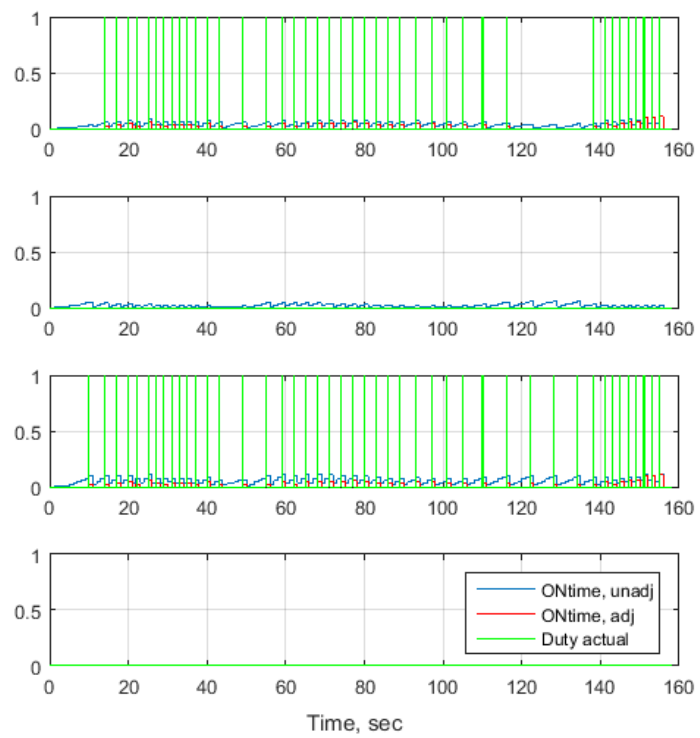

Figure 32. Nominal Successful Docking Example: Thruster on-time

Figure 33 presents the time history plot of the follower's attitude error in the X channel and the wheel speed. The attitude error stayed below 1 deg throughout the run until the magnetic forces and moments align the two test articles. The wheel speed stayed well within the limits but reached a greater maximum compare to the case where table uneveness is not included. 

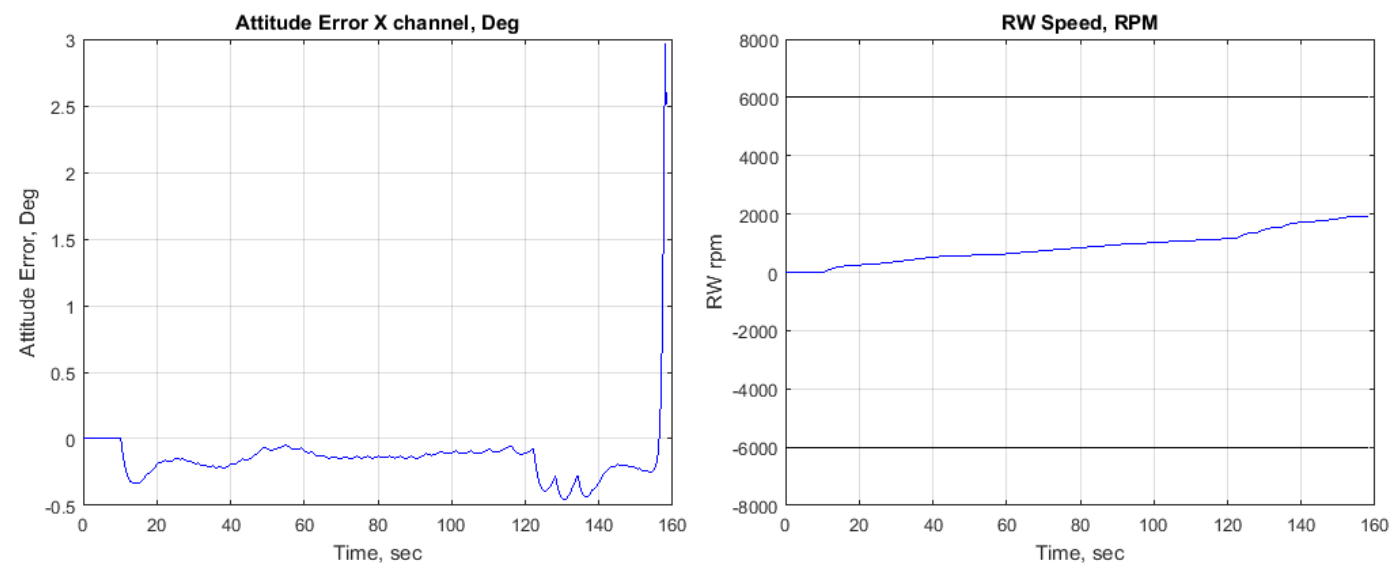

Figure 33. Nominal Successful Docking Example: Attitude Error and Reaction Wheel RPM

\section{Conclusion and Ongoing Work}

Autonomous rendezvous and docking of two CubeSats in space is a challenging GNC problem. The OnOrbit Autonomous Assembly of Nanosatellites (OAAN) team at NASA Langley Research Center along with Cornell University have developed a novel solution to this problem using permanent magnets which allow CubeSats to align and lock during the final docking phase. Extensive simulation results have demonstrated the proposed magnetic docking concept eliminates the need for high precision sensors and actuators. Work is ongoing to obtain experimental verification of the system on the flat floor facility. The team recently completed a series of attitude pointing tests that verified the performance of the reaction wheels and attitude control system.

\section{Acknowledgement}

The authors would to like thank the NASA Science Technology Mission Directorate (STMD) for funding to pursue this concept. The authors would also like to extend their gratitude to Dr. Jim Beaty, Robert McSwaine, RJ Bodkins, Dr. Mason Peck, Dr. Carlos Roithmayr, and Dr. Bandu Pamadi for their encouragement, mentoring and insight. The authors would like to thank former interns: Adam Weber, Dalton Kajuwa, Nick Harvey for their contributions on the characterization of the Piksi performance and development of the false lock algorithm, Nash Kocur, Jeremy Walko-Frankovic, Ivy Chang for their support on the development of the docking subsystem and low-drag base, Kristopher Stone, Shawn Stewart, and others for their support on the verification of the propulsion subsystem design.

\section{References}

${ }^{1}$ Bowen J, Tsuda, A. A. J. and Villa, M., "CubeSat Proximity Operations Demonstration Mission Update," IEEE, 2015.

${ }^{2}$ Nunes M, Sorenson, T. and Pilger, E., "On the development of a 6 DoF GNC framework for docking multiple small satellites," No. 2015-0868, AIAA SciTech, 2015.

${ }^{3}$ Underwood, J. and Pellegrino, C., "Auntonomous Assembly of a Reconfigurable Space Telescope - A CubeSat/Microsatellite Based Technology Demonstrator," No. SSC-VI-5, AIAA/USU Conference on Small Satellites, 2013.

${ }^{4}$ Underwood J, Pellegrino, C. L. V. B. C. and Baker, J., "Using CubeSat/micro-satellite technology to demonstrate the Autonomous Assembly of a Reconfigurable Space Telescope," Acta Astronautica, 2015.

${ }^{5}$ Pei J, Murchison, L. and et, "Autonomous Rendezvous and Docking of Two 3U Cubesats Using a Novel Permanent Magnet Docking Mechanism," No. 2016-1465, AIAA SciTech, 2016.

${ }^{6}$ Romano M, Friedman, D. and Shay, T., "Laboratory Experimentation of Autonomous Spacecract Approach and Docking to a Collaborative Target," Journal of Spacecraft and Rockets, Vol. 44, No. 1, Jan-Feb 2007.

${ }^{7}$ Sabatini R, Farnocchia, M. and Palmerini, G., "Design and Tests of a Frictionless 2D Platform for Studying Space Navigation and Control Subsytems," IEEE, 2012.

${ }^{8}$ El-Sheimy N, Hou, H. and Niu, X., "Analysis and Modeling of Inertial Sensors Using Allan Variance," IEEE Transactions of Instrumentation and Measurement, Vol. 57, No. 1, Jan 2008.

${ }^{9}$ Chen A., Saenz-Otero, A. H. M. and Miller, D., "Development of Formation Flight and Docking Algorithms Using the 
SPHERES Testbed," Small Sat Conference, 2001.

${ }^{10}$ SwiftNav, Piksi DataSheet, June 2013.

${ }^{11}$ Elandt R, Aslan, S. and Peck, M., "Six Axis thruster Characterization Technique for Small Satellites," TBD, 2017.

${ }^{12}$ Sidi, M., Spacecraft Dynamics and Control, Cambridge University Press, 5th ed., 2006.

${ }^{13}$ Mohiuddin, S. and Psiaki, M., "High-Altitude Satellite Relative Navigation Using Carrier-Phase Differential Global

Positioning System Techniques," Journal of Spacecraft and Rockets, Vol. 30, No. 5, Sept-Oct 2007.

${ }^{14}$ Leung, S. and Montebruck, O., "Real-Time Navigation of Formation-Flying Spacecraft using Global-Positioning-System Measurements," Journal of Guidance, Control, and Dynamics, Vol. 28, No. 2, Mar-Apr 2005.

${ }^{15}$ Bonin G, Roth, N. A. S. N. J. R. B. and Zee, R., "CanX-4 and CanX-5 Precision Formation Fight: Mission Accomplished!" No. SSC15-I-4, Small Sat Conference, 2015.

${ }^{16}$ Blue Canyon Technologies, XACT DataSheet, Feb 2015.

${ }^{17}$ Yung K, Landecker, P. and Vallani, D., "An Analytical Solution for the Force Between Two Magnetic Dipoles," Magnetic and Electrical Separation, Vol. 9, 1998, pp. 39-52.

${ }^{18}$ Landecker P, Vallani, D. and Yung, K., "An Analytical Solution for the Torque Between Two Magnetic Dipoles," Magnetic and Electrical Separation, Vol. 10, 1999, pp. 29-33.

${ }^{19}$ COMSOL Manual, 2016.

${ }^{20}$ NewWay Air Bearings, Air Bearing Application and Design Guide, Jan 2006.

${ }^{21}$ Olsson H, Astrom, K. and deWit, C., "Friction Models and Friction Compensation," 1997.

${ }^{22}$ NASA.gov, "Overview of the DART Mishap Investigation Results," 2006.

${ }^{23}$ Dennehy, C., "A Summary of Rendezvous, Proximity, Operations, Docking, and Undocking Lessons Learned from the Defense Adavance Research Project Agency Orbital Express Demonstration System Mission," No. NESC-RP-10-00628, NASATM, 2011.

${ }^{24}$ Durham, W., "Constrained Control Allocation: Three-Moment Problem," Journal of Guidance, Control, and Dynamics, Vol. 17, No. 2, Mar-Apr 1994.

${ }^{25}$ Orr, J. and Slegers, N., "High Efficiency Thrust Vector Control Allocation," Journal of Guidance, Control, and Dynamics, Vol. 37, No. 2, Mar-Apr 2014.

${ }^{26}$ Bodson, M., "Evaluation of Optimization Methods for Control Allocation," No. 2001-4223, AIAA, 2001.

${ }^{27}$ Oppenheimer, M. and Doman, D., "A Method for Including Control Effector Interaction in the Control Allocation," No. 2007-6418, AIAA, 2007.

${ }^{28}$ Clohessy, W. and Wiltshire, R., "Terminal Guidance System for Satellite Rendezvous," Journal of the Aerospace Sciences, Vol. 27, No. 9, 1960, pp. 653-658.

${ }^{29}$ Singla P, Subbarao, K. and Junkin, J., "Adaptive Output Feedback Control for Spacecraft Rendezvous and Docking Under Measurement Uncertainty," Journal of Guidance, Control, and Dynamics, Vol. 29, No. 4, July-August 2006.

${ }^{30}$ Kluever, C., "Feeback Control for Spacecraft Rendezvous and Docking," Journal of Guidance, Control, and Dynamics, Vol. 22, No. 4, July-August 1999.

${ }^{31}$ Tournes C, Shtessel, Y. and Foreman, D., Sliding Mode Control, InTech, 2011.

${ }^{32}$ Cairano S, Park, S. and Kolmanovsky, I., "Model Predictive Control Approach for Guidance of Spacecraft Rendezvous and Proximity Maneuvering," International Journal of Robust and Nonlinear Control, Vol. 22, May 2012, pp. 1398-1427.

${ }^{33}$ Crassidis, J., Optimal Estimation of Dynamic Systems, CRC Press, 2nd ed.

${ }^{34}$ Orr J, Wall, J. V. T. and Hall, C., "Stability and Flight Readiness of the SLS FCS with Adaptive Augmentation. Appendix D: Describing Function Analysis," NASA NESC Report, 2016. 